\title{
QUANTUM FIELDS AND MOTIVES
}

\author{
ALAIN CONNES AND MATILDE MARCOLLI
}

\section{Renormalization: PARTiCle Physics AND Hopf AlgEBras}

The main idea of renormalization is to correct the original Lagrangian of a quantum field theory by an infinite series of counterterms, labelled by the Feynman graphs that encode the combinatorics of the perturbative expansion of the theory. These counterterms have the effect of cancelling the ultraviolet divergences. Thus, in the procedure of perturbative renormalization, one introduces a counterterm $C(\Gamma)$ in the initial Lagrangian for every divergent one particle irreducible (1PI) Feynman diagram $\Gamma$. In the case of a renormalizable theory, all the necessary counterterms $C(\Gamma)$ can be obtained by modifying the numerical parameters that appear in the original Lagrangian. It is possible to modify these parameters and replace them by (divergent) series, since they are not observable, unlike actual physical quantities that have to be finite. One of the fundamental difficulties with any renormalization procedure is a systematic treatment of nested and overlapping divergences in multiloop diagrams.

\section{Dimensional regularization and minimal subtraction.}

One of the most effective renormalization techniques in quantum field theory is dimensional regularization (DimReg). It is widely used in perturbative calculations. It is based on an analytic continuation of Feynman diagrams to complex dimension $d \in \mathbb{C}$, in a neighborhood of the integral dimension $D$ at which UV divergences occur. For the complex dimension $d \rightarrow D$, the analytically continued integrals become singular and the expression admits a Laurent series expansion. Thus, within the framework of dimensional regularization, one can implement a renormalization by minimal subtraction, where the singular part of the Laurent series in $z=d-D$ is subtracted at each order in the loop expansion. This renormalization method (DimReg plus minimal subtraction) was developed by 't Hooft and Veltman 36, who applied it to one-loop calculations in scalar electrodynamics, discussed the problem of overlapping divergences, the Ward identities, the case of theories with fermions, and anomalies. The method has since been applied widely to perturbative calculations and it quickly became the standard regularization and renormalization method for nonabelian gauge theories and the standard model.

\section{Hopf algebras and the combinatorics of renormalization.}

The modern viewpoint on combinatorics, which unfolded in the 70s around the systematic and rigorous restructuring of its foundations advocated by Giancarlo Rota, showed how algebraic structures such as coalgebras, bialgebras, and Hopf algebras govern elaborate combinatorial phenomena (cf. [37, [55], [57]). The reason why such algebraic structures are naturally present lies in the fact that combinatorial objects tend to admit decomposition laws that reduce them to simpler pieces. Such 
laws are the source of coproduct rules. This principle was illustrated by many examples of incidence Hopf algebras arising from classes of graphs and matroids. The typical situation is families of finite graphs, closed under disjoint union and taking vertex induced subgraphs. These admit a coproduct of the form

$$
\Delta(\Gamma)=\sum_{W \subseteq V} \gamma_{W} \otimes \gamma_{V-W},
$$

where $V$ is the set of vertices of $\Gamma$ and $\gamma_{W}$ is the induced subgraph on a set of vertices $W \subseteq V$. Results from Hopf algebras in combinatorics were used, for instance, to study graph coloring problems.

During 1960s and 1970s Quantum Field Theory underwent a season of extraordinary theoretical development. The detailed knowledge theoretical physicists gained on the subject not only made it into something of an art, but refined it into a highly sophisticated instrument, capable of producing theoretical predictions that, to this day, match experiments with unprecedented precision. Renormalization plays a central role in the quantum theory of fields, in as it provides a consistent scheme for extracting from divergent expressions finite values that can be matched to physically observed quantities. Various renormalization schemes can be implemented (though here we will be concerned only with the "dimensional regularization and minimal subtraction" scheme described above). A renormalization scheme produces an extremely elaborate combinatorial recipe that accounts for structuring of subgraphs in a hierarchy of subdivergences and counterterms. Perturbative renormalization hence appears as one of the most elaborate combinatorial recipes imposed on us by nature.

Conceptually, the crucial issue in the combinatorics of perturbative renormalization is a scheme that accounts for subdivergences. This is achieved by a forest formula, which assigns to a graph $\Gamma$ a formal expression where the subdivergences have been dealt with through inductively defined counterterms. Subtraction of the corresponding counterterm from this formal expression finally yields the renormalized value for $\Gamma$. The definition of such formal expressions, as we discuss more in detail below, is related to decomposing a graph by extracting all possible divergent subgraphs $\gamma \subset \Gamma$ and considering corresponding graphs $\Gamma / \gamma$ obtained by collapsing $\gamma \subset \Gamma$ to a single vertex. Such decomposition is more complicated than those derived from incidence relations on graphs in many combinatorial problems, as it is adapted to the specific divergences of the physical theory and has to take into account other data like the distribution of external momenta. Still, one can see a suggestive analogy between the type of decomposition expressed by coproducts (1.1) and the decomposition

$$
\Delta(\Gamma)=\sum_{\gamma \subseteq \Gamma} \gamma \otimes \Gamma / \gamma
$$

in a sum over divergent subgraphs, which underlies the combinatorics of the forest formulae. It was the seminal work of Dirk Kreimer 43 in 1997 that paved the way to a conceptual mathematical formulation of perturbative renormalization, precisely by encoding the complicated combinatorics of forest formulae via a coproduct (1.2) and identifying the Hopf algebra that governed the renormalization procedure.

The extraction of a renormalized value from divergent Feynman integrals was related in [43] and [15] to the antipode in the Hopf algebra. However, the precise 
formula for the renormalized value given by the BPHZ procedure ([5], 34, 62] ) requires a further operation that twists the antipode, which, in this formulation, is not given directly in terms of the Hopf algebra structure. The main conceptual breakthrough in the understanding of the renormalization procedure, that fully reconciles it with the Hopf algebra structure, was then obtained in a later stage of development of the Connes-Kreimer theory of perturbative renormalization, [16], 17, where the BPHZ recursive formulae (see (1.3), (1.4), (1.5) below) are described in terms of the Birkhoff factorization of loops. We shall return to this point in Section 2

Given the state of affairs in combinatorics and in quantum field theory around the late '70s, it may seem surprising that the pursuit of a conceptual mathematical interpretation of the procedure of perturbative renormalization had to wait, as it did, until the late 1990s. One should keep in mind though that, during the 1970s, mathematicians and physicists were maximally apart. The tendency among physicists was to shift the emphasis heavily towards deriving efficient computational recipes at the expense of conceptual understanding, the latter being often dismissed as a mere exercise of pedantry. This position, though justifiable in developing a theoretical apparatus that could be continuously tested against experiments, had the effect of alienating mathematicians. While quantum mechanics stimulated and in turn benefited from a lot of advancements in modern mathematics (operator algebras, functional analysis), mathematicians shied away from quantum field theory, which they perceived as ill founded, riddled with inevitable divergences, and governed by obscure hands-on recipes. In more recent times, mathematicians and theoretical physicists found a renewed harmony of language, but this happened mostly in the context of string theory. This, however, bypasses many of the crucial problems posed by quantum field theory, by proposing a large restructuring of the foundations of high energy physics, which at present still awaits experimental confirmation. Thus, in particular, the new developments left pretty much untouched the problem of a conceptual understanding of the foundations of quantum field theory. Of course, there were at various times attempts to axiomatize quantum field theory in a way that would be palatable for mathematicians (algebraic and constructive quantum field theory, for instance). Such attempts unfortunately fell short of incorporating the full complexity of quantum field theory, especially with respect to the issue of perturbative renormalization. On the other hand, at present perturbative quantum field theory still remains the most accurate instrument for theoretical predictions in elementary particle physics and this impressive agreement between theory and nature calls for the best possible conceptual understanding of its foundational principles.

\section{Bogoliubov-Parasiuk preparation.}

The Bogoliubov-Parasiuk preparation, or BPHZ method (for Bogoliubov-ParasiukHepp-Zimmermann, [5], 34, 62] ) accounts for the presence of subdivergences, simultaneously taking care of the problem of the appearance of non-local terms and the organization of subdivergences via an inductive procedure. 
The BP preparation of a graph $\Gamma$, whose divergent integral we denote by $U(\Gamma)$, is given by the formal expression

$$
\bar{R}(\Gamma)=U(\Gamma)+\sum_{\gamma \subset \Gamma} C(\gamma) U(\Gamma / \gamma)
$$

where the sum is over divergent subgraphs. The $C(\gamma)$ are inductively defined counterterms, obtained (in the minimal subtraction scheme) by taking the pole part (here denoted by $T$ ) of the Laurent expansion in $z=d-D$ of a divergent expression,

$$
C(\Gamma)=-T(\bar{R}(\Gamma))=-T\left(U(\Gamma)+\sum_{\gamma \subset \Gamma} C(\gamma) U(\Gamma / \gamma)\right) .
$$

The renormalized value of $\Gamma$ is then given by the formula

$$
R(\Gamma)=\bar{R}(\Gamma)+C(\Gamma)=U(\Gamma)+C(\Gamma)+\sum_{\gamma \subset \Gamma} C(\gamma) U(\Gamma / \gamma) .
$$

Before continuing with the physics, we need to introduce some algebraic notions that will be useful in the rest of the paper.

\section{Hopf algebras and affine group schemes.}

While affine schemes are the geometric manifestation of commutative algebras, affine group schemes are the geometric counterpart of commutative Hopf algebras. The theory of affine group schemes is developed in SGA 3 [26].

Consider a commutative Hopf algebra $\mathcal{H}$ over a field $k$, which we assume here of characteristic zero. Thus, $\mathcal{H}$ is a commutative algebra with unit over $k$, endowed with a (not necessarily co-commutative) coproduct $\Delta: \mathcal{H} \rightarrow \mathcal{H} \otimes_{k} \mathcal{H}$, a counit $\varepsilon: \mathcal{H} \rightarrow k$, which are $k$-algebra morphisms and an antipode $S: \mathcal{H} \rightarrow \mathcal{H}$ which is a $k$-algebra antihomomorphism. These satisfy the "co-rules"

$$
\begin{array}{ll}
(\Delta \otimes i d) \Delta=(i d \otimes \Delta) \Delta & : \mathcal{H} \rightarrow \mathcal{H} \otimes_{k} \mathcal{H} \otimes_{k} \mathcal{H}, \\
(i d \otimes \varepsilon) \Delta=i d=(\varepsilon \otimes i d) \Delta & : \mathcal{H} \rightarrow \mathcal{H}, \\
m(i d \otimes S) \Delta=m(S \otimes i d) \Delta=1 \varepsilon & : \mathcal{H} \rightarrow \mathcal{H},
\end{array}
$$

where we used $m$ to denote multiplication in $\mathcal{H}$.

One then lets $G=\operatorname{Spec} \mathcal{H}$ be the set of prime ideals of the commutative $k$-algebra $\mathcal{H}$, with the Zariski topology. The Zariski topology is too coarse to fully recover the "algebra of coordinates" $\mathcal{H}$ from the topological space $\operatorname{Spec}(\mathcal{H})$, but one recovers it through the data of the structure sheaf, i.e. by considering global sections of the "sheaf of functions" on $\operatorname{Spec}(\mathcal{H})$.

Since $\mathcal{H}$ is a commutative $k$-algebra, $G=\operatorname{Spec}(\mathcal{H})$ is an affine scheme over $k$, while the additional structure given by the co-rules (1.6) endow $G=\operatorname{Spec}(\mathcal{H})$ with a product operation, a unit, and an inverse.

More precisely, one can view such $G$ as a functor that associates to any unital commutative algebra $A$ over $k$ a group $G(A)$, whose elements are the $k$-algebra homomorphisms

$$
\phi: \mathcal{H} \rightarrow A, \quad \phi(x y)=\phi(x) \phi(y), \quad \forall x, y \in \mathcal{H}, \quad \phi(1)=1 .
$$


The product in $G(A)$ is given as the dual of the coproduct, by

$$
\phi_{1} \star \phi_{2}(x)=\left\langle\phi_{1} \otimes \phi_{2}, \Delta(x)\right\rangle .
$$

The inverse and the unit of $G(A)$ are determined by the antipode and the co-unit of $\mathcal{H}$. The co-rules imply that these operations define a group structure on $G(A)$. The resulting covariant functor

$$
A \rightarrow G(A)
$$

from commutative algebras to groups is representable (in fact by $\mathcal{H}$ ). The functor $G$ obtained in this way is called an affine group scheme. Conversely, any covariant representable functor from the category of commutative algebras over $k$ to groups, is an affine group scheme $G$, represented by a commutative Hopf algebra, uniquely determined up to canonical isomorphism.

Some simple examples of affine group schemes:

- The multiplicative group $G=\mathbb{G}_{m}$ is the affine group scheme obtained from the Hopf algebra $\mathcal{H}=k\left[t, t^{-1}\right]$ with coproduct $\Delta(t)=t \otimes t$.

- The additive group $G=\mathbb{G}_{a}$ corresponds to the Hopf algebra $\mathcal{H}=k[t]$ with coproduct $\Delta(t)=t \otimes 1+1 \otimes t$.

- The affine group scheme $G=\mathrm{GL}_{n}$ corresponds to the Hopf algebra

$$
\mathcal{H}=k\left[x_{i, j}, t\right]_{i, j=1, \ldots, n} / \operatorname{det}\left(x_{i, j}\right) t-1,
$$

with coproduct $\Delta\left(x_{i, j}\right)=\sum_{k} x_{i, k} \otimes x_{k, j}$.

The latter example is quite general. In fact, if $\mathcal{H}$ is finitely generated as an algebra over $k$, then the corresponding affine group scheme $G$ is a linear algebraic group over $k$, and can be embedded as a Zariski closed subset in some $\mathrm{GL}_{n}$. Moreover, in the more general case, one can find a collection $\mathcal{H}_{i} \subset \mathcal{H}$ of finitely generated algebras over $k$ such that $\Delta\left(\mathcal{H}_{i}\right) \subset \mathcal{H}_{i} \otimes \mathcal{H}_{i}, S\left(\mathcal{H}_{i}\right) \subset \mathcal{H}_{i}$, for all $i$, and such that, for all $i, j$ there exists a $k$ with $\mathcal{H}_{i} \cup \mathcal{H}_{j} \subset \mathcal{H}_{k}$, and $\mathcal{H}=\cup_{i} \mathcal{H}_{i}$. In this case, one obtains linear algebraic groups $G_{i}=\operatorname{Spec}\left(\mathcal{H}_{i}\right)$ such that

$$
G=\lim _{i} G_{i} .
$$

Thus, in general, an affine group scheme is a projective limit of linear algebraic groups. If the $G_{i}$ are unipotent, then $G$ is a pro-unipotent affine group scheme.

The Lie algebra $\mathfrak{g}(k)=\operatorname{Lie} G(k)$ is given by the set of linear maps $L: \mathcal{H} \rightarrow k$ satisfying

$$
L(X Y)=L(X) \varepsilon(Y)+\varepsilon(X) L(Y), \quad \forall X, Y \in \mathcal{H},
$$

where $\varepsilon$ is the co-unit of $\mathcal{H}$, playing the role of the unit in the dual algebra. Equivalently, $\mathfrak{g}=$ Lie $G$ is a covariant functor

$$
A \rightarrow \mathfrak{g}(A),
$$

from commutative $k$-algebras to Lie algebras, where $\mathfrak{g}(A)$ is the Lie algebra of linear maps $L: \mathcal{H} \rightarrow A$ satisfying (1.9). 


\section{Hopf algebra of Feynman graphs and diffeographisms.}

The Kreimer Hopf algebra of [43] is based on rooted trees, which organize the hierarchy of subdivergences in a given graph. The Hopf algebra depends on the particular physical theory $\mathcal{T}$ through the use of trees whose vertices are decorated by the divergence free Feynman graphs of the theory ( $c f$. [43] [15]). In the work of Connes-Kreimer [16] this Hopf algebra was refined to a Hopf algebra $\mathcal{H}(\mathcal{T})$, also dependent on the physical theory $\mathcal{T}$ by construction, which is directly defined in terms of Feynman graphs.

The CK Hopf algebra is the free commutative algebra over $k=\mathbb{C}$ generated by one particle irreducible (1PI) graphs $\Gamma\left(p_{1}, \ldots, p_{n}\right)$, where $\Gamma$ is not a tree. A graph $\Gamma$ is $1 \mathrm{PI}$ if it cannot be disconnected by the removal of a single edge. Here one considers graphs endowed with external momenta $\left(p_{1}, \ldots, p_{n}\right)$. To account for this external structure one considers distributions $\sigma \in C_{c}^{-\infty}\left(E_{\Gamma}\right)$ for

$$
E_{\Gamma}=\left\{\left(p_{i}\right)_{i=1, \ldots, N} ; \sum p_{i}=0\right\},
$$

and the symmetric algebra $\mathcal{H}=S\left(C_{c}^{-\infty}\left(\cup E_{\Gamma}\right)\right)$, with $\cup E_{\Gamma}$ the disjoint union.

The coproduct is given by a formula that reflects the BP preparation (1.3), namely, it is given on generators by the expression

$$
\Delta(\Gamma)=\Gamma \otimes 1+1 \otimes \Gamma+\sum_{\gamma \subset \Gamma} \gamma_{(i)} \otimes \Gamma / \gamma_{(i)} .
$$

Here the sum is over divergent subgraphs $\gamma \subset \Gamma$ and $\Gamma / \gamma$ denotes the graph obtained by contracting $\gamma$ to a single vertex. In (1.11) the notation $\gamma_{(i)}$ accounts for the fact that one has to specify how to assign the external structure to $\gamma$, depending on the type of the corresponding vertex in $\Gamma / \gamma_{(i)}, c f$. [16].

Up to passing to the Hopf subalgebra constructed on 1PI graphs with fixed external structure, one can reduce to a Hopf algebra $\mathcal{H}(\mathcal{T})$ that is finite dimensional in each degree, where the degree is defined on 1PI graphs by the loop number. There is an affine group scheme associated to this Hopf algebra $\mathcal{H}(\mathcal{T})$. This is called the group of diffeographisms $G=\operatorname{Difg}(\mathcal{T})$ of the physical theory. It is a pro-unipotent affine group scheme.

The reason for the terminology lies in the fact that $\operatorname{Difg}(\mathcal{T})$ has a close relation to the group of formal diffeomorphisms of the complexified coupling constants of the theory. In the simplest case this group is the group Diff($(\mathbb{C})$ of formal diffeomorphisms of the complex line tangent to the identity. The latter corresponds to the Hopf algebra $\mathcal{H}_{\text {diff }}$ whose generators $a_{n}$ are obtained by writing formal diffeomorphisms as $\varphi(x)=x+\sum_{n \geq 2} a_{n}(\varphi) x^{n}$, and with coproduct $\left\langle\Delta a_{n}, \varphi_{1} \otimes \varphi_{2}\right\rangle=a_{n}\left(\varphi_{2} \circ \varphi_{1}\right)$. A Hopf algebra homomorphism is obtained by writing the effective coupling constant as a formal power series $g_{\mathrm{eff}}(g)=g+\sum_{n>2} \alpha_{n} g^{n}$, where all the coefficients $\alpha_{n}$ are finite linear combinations of products of graphs, $\alpha_{n} \in \mathcal{H}$, for all $n \geq 1$ and mapping $a_{n} \mapsto \alpha_{n}, c f$. [16.

\section{BIRKHOFF FACTORIZATION AND RENORMALIZATION}

Suppose given a complex Lie group $G(\mathbb{C})$ and a smooth simple curve $C \subset \mathbb{P}^{1}(\mathbb{C})$, with $C^{ \pm}$the two complementary regions, with $\infty \in C^{-}$. For a given loop $\gamma: C \rightarrow$ 
$G(\mathbb{C})$, the problem of Birkhoff factorization asks whether there exist holomorphic maps $\gamma_{ \pm}: C_{ \pm} \rightarrow G(\mathbb{C})$, such that

$$
\gamma(z)=\gamma_{-}(z)^{-1} \gamma_{+}(z) \quad z \in C .
$$

This procedure of factorization of Lie group valued loops became well known in algebraic geometry because of its use in the Grothendieck-Birkhoff decomposition 31 of holomorphic vector bundles on the sphere $\mathbb{P}^{1}(\mathbb{C})$. In this case, the Lie group is $\mathrm{GL}_{n}(\mathbb{C})$ and a weaker form of (2.1) holds, whereby loops factor as

$$
\gamma(z)=\gamma_{-}(z)^{-1} \lambda(z) \gamma_{+}(z),
$$

where $\lambda(z)$ is a diagonal matrix with entries $\left(z^{k_{1}}, z^{k_{2}}, \cdots, z^{k_{n}}\right)$. The GrothendieckBirkhoff decomposition hence states that a holomorphic vector bundle on $\mathbb{P}^{1}(\mathbb{C})$ can be described as $E=L^{k_{1}} \oplus \ldots \oplus L^{k_{n}}$, where the line bundles $L^{k_{i}}$ have Chern class $c_{1}\left(L^{k_{i}}\right)=k_{i}$. This corresponds to the Birkhoff decomposition (2.1) when $c_{1}\left(L^{k_{i}}\right)=0$.

From a more analytic viewpoint (cf. e.g. 7]), the Birkhoff factorizations (2.1) or (2.2) can be viewed as a (homogeneous) transmission problem, which can be formulated in terms of systems of singular integral equations, with various regularity assumptions. Such transmission problems can be recast in the context of the theory of Fredholm pairs, obtained by considering the spaces of boundary values, on a simple closed curve $C$, of sections of holomorphic vector bundles on $\mathbb{P}^{1}(\mathbb{C})$.

\section{BPHZ as a Birkhoff factorization.}

One of the key results of the Connes-Kreimer theory of perturbative renormalization [16 [17 is a reformulation of the BPHZ procedure as a Birkhoff factorization in the pro-unipotent Lie group $G(\mathbb{C})$ associated to the affine group scheme $G=\operatorname{Difg}(\mathcal{T})$.

Unlike the case of $\mathrm{GL}_{n}$, where the Birkhoff decomposition (2.1) only holds when $k_{i}=0$, in the case of interest for renormalization one always has a factorization (2.1). This follows from a result of Connes-Kreimer, which we recall in Proposition 2.1 below. For the general case where $G$ is the pro-unipotent affine group scheme of a Hopf algebra that is graded in positive degree and connected, the result shows that a factorization of the form (2.1) always exists. The result, in fact, provides an explicit recursive formula, in Hopf algebra terms, which determines both terms in the factorization.

In this setup, the Lie group $G(\mathbb{C})$ is the set of complex points of an affine group scheme $G$, whose commutative Hopf algebra $\mathcal{H}$ is graded in positive degrees $\mathcal{H}=$ $\cup_{k} \mathcal{H}_{k}$ and connected (i.e. the only elements of degree 0 in $\mathcal{H}$ are the scalars).

We let $K=\mathbb{C}(\{z\})$ be the field of Laurent series convergent in some neighborhood of the origin (i.e. germs of meromorphic functions at the origin) and $\varnothing=\mathbb{C}\{z\}$ be the ring of convergent power series, and we let $\mathcal{Q}=z^{-1} \mathbb{C}\left(\left[z^{-1}\right]\right)$, with $\tilde{\mathcal{Q}}=\mathbb{C}\left(\left[z^{-1}\right]\right)$ the corresponding unital ring. Then a loop $\gamma: C \rightarrow G$, for $C$ an infinitesimal circle around the origin, is equivalently described by a homomorphism $\phi: \mathcal{H} \rightarrow K$, i.e. by a point in $G(K)$. Because the group structure on $G$ corresponds to the co-rules of the Hopf algebra $\mathcal{H}$, the product of loops $\gamma(z)=\gamma_{1}(z) \gamma_{2}(z)$, for $z \in C$, corresponds to $\phi=\phi_{1} \star \phi_{2}$ (dual to the coproduct in $\mathcal{H}$ ) and the inverse $z \mapsto \gamma(z)^{-1}$ to the antipode $\phi \circ S$. 
For $z=0 \in C^{+}$, the condition that the loop $\gamma$ extends to a holomorphic function $\gamma: P_{1}(\mathbb{C}) \backslash\{0\} \rightarrow G$ is equivalent to the condition that the homomorphism $\phi$ lies in $G(\tilde{\mathcal{Q}})=\{\phi, \phi(\mathcal{H}) \subset \tilde{\mathcal{Q}}\}$, while the condition that $\gamma(0)$ is finite translates in the condition that $\phi$ belongs to $G(\varnothing)=\{\phi, \phi(\mathcal{H}) \subset \varnothing\}$. The normalization condition $\gamma(\infty)=1$ translates algebraically into the condition $\varepsilon_{-} \circ \phi=\varepsilon$, where $\varepsilon_{-}$is the augmentation in the ring $\tilde{\mathcal{Q}}$ and $\varepsilon$ is the augmentation (co-unit) of $\mathcal{H}$. This dictionary shows how interpreting affine group schemes as functors of unital commutative algebras to groups provides a very convenient language in which to reformulate the problem of Birkhoff factorization.

Proposition 2.1. (16]) Let $\mathcal{H}$ be a Hopf algebra that is graded in positive degree and connected, and $G$ the corresponding affine group scheme. Then any loop $\gamma$ : $C \rightarrow G(\mathbb{C})$ admits a Birkhoff factorization (2.1). An explicit recursive formula for the factorization is given, in terms of the corresponding homomorphism $\phi: \mathcal{H} \rightarrow$ $\mathbb{C}(\{z\})$, by the expressions

$$
\phi_{-}(X)=-T\left(\phi(X)+\sum \phi_{-}\left(X^{\prime}\right) \phi\left(X^{\prime \prime}\right)\right)
$$

and

$$
\phi_{+}(X)=\phi(X)+\phi_{-}(X)+\sum \phi_{-}\left(X^{\prime}\right) \phi\left(X^{\prime \prime}\right),
$$

where $T$ is the projection along $\varnothing$ to the augmentation ideal of $\tilde{\mathcal{Q}}$ (taking the pole part), and $X^{\prime}$ and $X^{\prime \prime}$ denote the terms of lower degree in the coproduct $\Delta(X)=$ $X \otimes 1+1 \otimes X+\sum X^{\prime} \otimes X^{\prime \prime}$, for $X \in \mathcal{H}$.

Applied to the Hopf algebra $\mathcal{H}(\mathcal{T})$ of Feynman graphs, with $G=\operatorname{Difg}(\mathcal{T})$, the formulae (2.3) and (2.4) yield the counterterms (1.4) and the renormalized values (1.5) in the BPHZ renormalization procedure.

\section{Mass parameter, counterterms, and the renormalization group.}

In DimReg, when analytically continuing the Feynman graphs to complex dimension, in order to preserve the dimensionality of the integrand in physical units, one needs to replace the momentum space integration $d^{D-z} k$ by $\mu^{z} d^{D-z} k$, where $\mu$ is a mass parameter, so that the resulting quantity has the correct dimensionality of (mass $)^{D}$. This introduces a dependence on the parameter $\mu$ in the loop $\gamma_{\mu}(z)$ describing the unrenormalized theory. The behavior of a renormalizable theory under rescaling of the mass parameter $\mu \mapsto e^{t} \mu$, for $t \in \mathbb{R}$, was analyzed in [35.

An important result, which will play a crucial role in our geometric formulation in Section [5 is that the counterterms do not depend on the mass parameter $\mu$ (cf. [13] $\S 5.8$ and $\S 7.1)$. This result translates in terms of the Birkhoff factorization to the condition that the negative part $\gamma_{\mu^{-}}(z)$ of the factorization $\gamma_{\mu}(z)=\gamma_{\mu^{-}}(z)^{-1} \gamma_{\mu^{+}}(z)$ satisfies

$$
\frac{\partial}{\partial \mu} \gamma_{\mu^{-}}(z)=0
$$

The effect of scaling the mass parameter on the loop $\gamma_{\mu}(z)$ is instead described by the action of the 1-parameter group of automorphisms generated by the grading by loop number. Namely, if $\theta_{t}$ denotes the 1-parameter group with infinitesimal generator $\left.\frac{d}{d t} \theta_{t}\right|_{t=0}=Y$, where $Y$ is the grading by loop number, we have

$$
\gamma_{e^{t} \mu}(z)=\theta_{t z}\left(\gamma_{\mu}(z)\right), \quad \forall t \in \mathbb{R}
$$


and for all $z$ in an infinitesimal punctured neighborhood $\Delta^{*}$ of the origin $z=$ $d-D=0$.

A well known but unpublished result of 't Hooft shows that the counterterms in a renormalizable quantum field theory can be reconstructed from the beta function of the theory. In the context of the Connes-Kreimer theory of perturbative renormalization, this can be seen in the following way.

The beta function here is lifted from the space of the coupling constants of the theory to the group of Diffeographisms, namely, it can be regarded as an element in the Lie algebra $\operatorname{Lie} G$ satisfying

$$
\beta=Y \operatorname{Res} \gamma,
$$

where $Y$ is the grading by loop number, and the residue of $\gamma$ is given by

$$
\operatorname{Res}_{z=0} \gamma=-\left(\frac{\partial}{\partial u} \gamma_{-}\left(\frac{1}{u}\right)\right)_{u=0} .
$$

The beta function is the infinitesimal generator $\beta=\left.\frac{d}{d t} \mathbf{r g}_{t}\right|_{t=0}$ of the renormalization group

$$
\mathbf{r g}_{t}=\lim _{z \rightarrow 0} \gamma_{-}(z) \theta_{t z}\left(\gamma_{-}(z)^{-1}\right)
$$

Correspondingly, the renormalized value, that is, the finite value $\gamma_{\mu}^{+}(0)$ of the Birkhoff decomposition satisfies the equation

$$
\mu \frac{\partial}{\partial \mu} \gamma_{\mu}^{+}(0)=\beta \gamma_{\mu}^{+}(0) .
$$

A strong form of the 't Hooft relations, deriving the counterterms from the beta function, is given by the following result.

Proposition 2.2. (17]) The negative part of the Birkhoff factorization $\gamma_{-}(z)$ satisfies

$$
\gamma_{-}(z)^{-1}=1+\sum_{n=1}^{\infty} \frac{d_{n}}{z^{n}},
$$

where the coefficients $d_{n}$ are given by iterated integrals

$$
d_{n}=\int_{s_{1} \geq s_{2} \geq \cdots \geq s_{n} \geq 0} \theta_{-s_{1}}(\beta) \theta_{-s_{2}}(\beta) \ldots \theta_{-s_{n}}(\beta) d s_{1} \ldots d s_{n} .
$$

The result can be formulated ( $c f$. [17]) as a scattering formula

$$
\gamma_{-}(z)=\lim _{t \rightarrow \infty} e^{-t\left(\frac{\beta}{z}+Z_{0}\right)} e^{t Z_{0}},
$$

where $Z_{0}$ is the additional generator of the Lie algebra of $G \rtimes_{\theta} \mathbb{G}_{a}$, satisfying

$$
\left[Z_{0}, X\right]=Y(X) \quad \forall X \in \operatorname{Lie} G .
$$

This form of the 't Hooft relations and the explicit formula (2.12) in terms of iterated integrals are the starting point for our formulation of perturbative renormalization in terms of the Riemann-Hilbert correspondence and for the relation to motivic Galois theory.

Before continuing with a more detailed account of these topics, we give an introductory tour of some ideas underlying the theory of motives and the Riemann-Hilbert correspondence, that we will need in order to introduce the main result of [18]. 


\section{The Yoga OF MOtives: COHOMOLOGIES AS AVATARS}

There are several possible cohomology theories that can be applied to algebraic varieties. Over a field $k$ of characteristic zero one has de Rham cohomology $H_{d R}(X)=\mathbb{H}^{\cdot}\left(X, \Omega_{X}\right)$, defined in terms of sheaves of differential forms, and Betti cohomology $H_{B}^{-}(X, \mathbb{Q})$, which is a version of singular homology for $\sigma X(\mathbb{C})$, for an embedding $\sigma: k \hookrightarrow \mathbb{C}$. These are related by the periods isomorphism

$$
H_{d R}^{i}(X, k) \otimes_{\sigma} \mathbb{C} \cong H_{B}^{i}(X, \mathbb{Q}) \otimes_{\mathbb{Q}} \mathbb{C} .
$$

Over a perfect field of positive characteristic there is also crystalline cohomology, while in all characteristics one can consider étale cohomology given by finite dimensional $\mathbb{Q}_{\ell}$-vector spaces $H_{e t}^{i}\left(\bar{X}, \mathbb{Q}_{\ell}\right)$, where $\bar{X}$ is obtained by extension of scalars to an algebraic closure $\bar{k}$, and $\ell \neq$ char $k$. In the smooth projective case, these have the expected properties of Poincaré duality, Künneth isomorphisms, etc. Moreover, étale cohomology provides interesting $\ell$-adic representations of $\operatorname{Gal}(\bar{k} / k)$. There are comparison isomorphisms

$$
H_{B}^{i}(X, \mathbb{Q}) \otimes_{\mathbb{Q}} \mathbb{Q}_{\ell} \cong H_{e t}^{i}\left(\bar{X}, \mathbb{Q}_{\ell}\right) .
$$

The natural question is then what type of information, such as maps or operations on one cohomology, can be transferred to the other ones. This gave rise to the idea, proposed by Grothendieck, of the existence of a "universal cohomology theory" with realization functors to all the known cohomology theories for algebraic varieties. He called this the theory of motives.

A metaphor 33] justifying the terminology is provided by music scores, some of which (such as Bach's "Art of the fugue") are not written for any particular instrument. They are just the motive, which in turn can be realized on different musical instruments. Another powerful metaphor is provided by the notion of avatar in Hindu philosophy, which expresses the idea of a single entity manifesting itself in manifold incarnations (the ten avatars of Vishnu).

We will present here only a very short overview of some ideas and results about motives, following [23, 48, [58, and 4, 24, 30, 46. We start first by recalling some general algebraic formalism we will need in the following.

\section{Tannakian categories.}

The basis for a Galois theory of motives lies in a suitable categorical formalism. This was first proposed by Grothendieck, who used the term Galois-Poincaré categories (or rigid tensor categories), and was then developed by Saavedra [56], who introduced the now currently adopted terminology of Tannakian categories, and by Deligne-Milne 25] ( $c f$. also the more recent 22]).

It is well known that there are many deep analogies between the theory of coverings of topological spaces and Galois theory. The analogy starts with the observation that, in cases where the covering spaces are defined by algebraic equations, the Galois symmetries of the equation actually correspond to deck transformations of the covering space.

Grothendieck brought this initial simple analogy to far reaching consequences. He developed a common formalism where fundamental groups (of a space, a scheme, or much more generally a topos) and Galois groups both fit naturally. The idea is that, in this very general setting, the group always arises as the group of automorphisms 
of a fiber functor on a suitable "category of coverings". The theory of the (profinite) fundamental groups is based on the existence of a fiber functor from a certain category $\mathcal{C}$ of finite étale covers of a connected scheme $S$, with values in finite sets. Then such functor $\omega$ yields an equivalence of categories between $\mathcal{C}$ and $G$ sets for $G=\operatorname{Aut}(\omega)$ a pro-finite group. This yields a profinite completion of the fundamental group. For $S=\operatorname{Spec}(K)$, it gives Galois theory, thus effectively bringing fundamental groups and Galois groups within the same general formalism.

This is the fundamental idea that guided the development of a motivic Galois theory. The latter appeared as a "linear" version of the general formalism described above, where the fiber functor is a faithful and exact tensor functor with values in vector spaces (instead of finite sets), and the Galois group is the affine group scheme $G=\operatorname{Aut}^{\otimes}(\omega)$.

More precisely, an abelian category is a category to which the tools of homological algebra apply, that is, a category where the sets of morphisms are abelian groups, there are products and coproducts, kernels and cokernels always exist and satisfy the same basic rules as in the category of modules over a ring. A tensor category over a field $k$ of characteristic zero is a $k$-linear abelian category $\mathbb{T}$ endowed with a tensor functor $\otimes: \mathbb{T} \times \mathbb{T} \rightarrow \mathbb{T}$ satisfying associativity and commutativity laws defined by functorial isomorphisms, and with a unit object. Moreover, for each object $X$, there exists a dual $X^{\vee}$ and maps $e v: X \otimes X^{\vee} \rightarrow 1$ and $\delta: 1 \rightarrow X \otimes X^{\vee}$, such that the composites $(e v \otimes 1) \circ(1 \otimes \delta)$ and $(1 \otimes e v) \circ(\delta \otimes 1)$ are the identity, with an identification $k \simeq \operatorname{End}(1)$.

A Tannakian category $\mathbb{T}$ over $k$ is a tensor category endowed with a fiber functor, namely a functor $\omega$ to finite dimensional vector spaces Vect ${ }_{K}$, for $K$ an extension of $k$, satisfying $\omega(X) \otimes \omega(Y) \simeq \omega(X \otimes Y)$ compatibly with associativity commutativity and unit. (A more general formulation can be given with values in locally free sheaves over a scheme, see [22]). A neutral Tannakian category $\mathbb{T}$ has a Vect $\mathrm{k}^{-}$ valued fiber functor $\omega$. In this case, the main result is that the fiber functor $\omega$ induces an equivalence of categories between $\mathbb{T}$ and the category $\operatorname{Rep}_{G}$ of finite dimensional linear representations of a uniquely determined affine group scheme $G=\operatorname{Aut}^{\otimes}(\omega)$, given by the automorphisms of the fiber functor.

A $k$-linear abelian category $\mathbb{T}$ is semi-simple if there exists $A \subset O b(\mathbb{T})$ such that all objects $X$ in $A$ are simple (namely $\operatorname{Hom}(X, X) \simeq k$ ), with $\operatorname{Hom}(X, Y)=0$ for $X \neq Y$ in $A$, and such that every object of $\mathbb{T}$ is isomorphic to a direct sum of objects in A. The affine group scheme $G$ of a neutral Tannakian category is pro-reductive if and only if the category is semi-simple.

As an example, one can consider the category of finite dimensional complex linear representations of a group. It is not hard to see what is in this case the structure of neutral Tannakian category, with fiber functor the forgetful functor to complex vector spaces. The affine group scheme determined by this neutral Tannakian category is called the "algebraic hull" of the group. In the case of the group $\mathbb{Z}$, the algebraic hull is an extension of $\hat{\mathbb{Z}}$, with the corresponding commutative Hopf algebra given by $\mathcal{H}=\mathbb{C}[e(q), t]$, for $q \in \mathbb{C} / \mathbb{Z}$, with the relations $e\left(q_{1}+q_{2}\right)=$ $e\left(q_{1}\right) e\left(q_{2}\right)$ and the coproduct $\Delta(e(q))=e(q) \otimes e(q)$ and $\Delta(t)=t \otimes 1+1 \otimes t$.

The non-neutral case where $\omega$ takes values in $\operatorname{Vect}_{K}$ for some extension of $k$, or the more general case of locally free sheaves over a scheme, can also be identified with a category of representations, but now the group $G$ is replaced by a groupoid 
(Grothendieck's Galois-Poincaré groupoid). This corresponds to the fact that, even in the original case of fundamental groups of topological spaces, it is more natural to work with the notion of fundamental groupoid, rather than with the base point dependent fundamental group. For our purposes, however, it will be sufficient to work with the more restrictive notion of neutral Tannakian category.

\section{Gauge groups and categories.}

In 22], $\S 7$, Deligne gives a characterization of Tannakian categories, over a field $k$ of characteristic zero, as tensor categories where the dimensions are positive integers. The dimension of $X \in \mathbb{T}$ is defined in this context as $\operatorname{Tr}\left(1_{X}\right)$, where $\operatorname{Tr}(f)=e v \circ \delta(f)$.

This characterization is very close to results developed via different techniques by Doplicher and Roberts in the context of algebraic quantum field theory, [27]. Their motivation was to derive the existence of a global compact gauge group, given the local observables of the theory. The group is obtained from a monoidal $C^{*}$ category where the objects are endomorphisms of certain unital $C^{*}$-algebras and the arrows are intertwining operators between these endomorphisms. They obtain a characterization of those monoidal $C^{*}$-categories that are equivalent to the category of finite dimensional continuous unitary representations of a compact group, unique up to isomorphism. Though the context and the techniques employed in the proof are different, the result has a flavor similar to the relation between Tannakian categories and affine group schemes. In their proof, a characterization analogous to the one of 22], $\S 7$ of the integer dimensions also plays an important role.

\section{Pure and mixed motives.}

The first constructions of a category of motives proposed by Grothendieck covers the case of smooth projective varieties. The corresponding motives form a $\mathbb{Q}$ linear abelian category $\mathcal{M}_{\text {pure }}(k)$ of pure motives. There is a contravariant functor assigning a motive to a variety

$$
X \mapsto h(X)=\oplus_{i} h^{i}(X) .
$$

If $h^{j}=0$, for all $j \neq i$, the motive is pure of weight $i$. This way a pure motive can be thought of as a "direct summand of an algebraic variety". The morphisms $\operatorname{Hom}(X, Y)$ in the category of motives are given by correspondences, namely algebraic cycles in the product $X \times Y$ of codimension equal to the dimension of $X$, modulo a suitable equivalence relation. Different choices of the notion of equivalence for algebraic cycles produce variants of the theory, ranging from the coarsest numerical equivalence to the finest rational equivalence (Chow groups). The objects of the category also include kernels of projectors, namely of idempotents in $\operatorname{Hom}(X, Y)$. Thus, for $p=p^{2} \in \operatorname{End}(X)$ and $q=q^{2} \in \operatorname{End}(Y)$, one takes $\operatorname{Hom}((X, p),(Y, q))=q \operatorname{Hom}(X, Y) p$.

One also adds to the objects the Tate motive $\mathbb{Q}(1)$, which is the inverse of $h^{2}\left(\mathbb{P}^{1}\right)$. This is a pure motive of weight -2 . The category is endowed with a tensor product $\otimes$ and a unit $\mathbb{Q}(0)=h(p t)$. The Tate objects $\mathbb{Q}(n)$ satisfy the rule $\mathbb{Q}(n+m) \cong$ $\mathbb{Q}(n) \otimes \mathbb{Q}(m)$.

Grothendieck formulated a set of standard conjectures about pure motives, which are at present still unproven. Assuming the standard conjectures, the category of pure motives is a neutral Tannakian category, with fiber functors given by Betti 
cohomology (characteristic zero case). Thus, the category of pure motives is equivalent to the category of representations $R e p_{G}$ of an affine group scheme $G$. This group is called the motivic Galois group. The category of pure motives is conjecturally semi-simple, hence for pure motives $G$ is pro-reductive.

When one considers certain subcategories of the category of motives, one obtains a corresponding Galois group, which is a quotient of the original $G$. For instance, if the subcategory is generated by a single $X$, one obtains a quotient $G_{X}$, whose identity component is the Mumford-Tate group of $X$. The subcategory of pure Tate motives, generated by $\mathbb{Q}(1)$ has as motivic Galois group the multiplicative group $\mathbb{G}_{m}$.

Some of the first unconditional results about motives were obtained in 48. In general, a serious technical obstacle in the development of the theory of motives, which accounts for the fact that, decades after its conception, the theory is still largely depending on conjectures, is the fact that not enough is known about algebraic cycles. The situation gets even more complicated when one wishes to consider more general algebraic varieties, which need not be smooth projective. This leads to the notion of mixed motives with $\mathcal{M}_{\text {pure }}(k) \subset \mathcal{M}_{\text {mix }}(k)$.

Over a field of characteristic zero (where one has resolution of singularities), one can always write such $X$ as a disjoint union of $X_{i}-D_{i}$, where the $X_{i}$ are smooth projective and the $D_{i}$ are lower dimensional. Thus, one can assign to $X$ a virtual object in a suitable Grothendieck group of algebraic varieties; however, if one wants a theory that satisfies the main requirements of a category of motives, including the fact of providing a universal cohomology theory (via the Ext functors), the construction of such a category of mixed motives remains a difficult task.

The main properties for a category of mixed motives are that it should be a $\mathbb{Q}$ linear tensor category containing the Tate objects $\mathbb{Q}(n)$ with the usual properties, endowed with a functor $X \mapsto h(X)$ that assigns motives to algebraic varieties, with properties like Künneth isomorphisms. Moreover, the Ext functors in this category of mixed motives define a "motivic cohomology"

$$
E_{2}^{i, j}=\operatorname{Ext}^{i}\left(\mathbb{Q}(0), h^{j}(X) \otimes \mathbb{Q}(n)\right) \Rightarrow H_{m o t}^{i+j}(X, \mathbb{Q}(n)) .
$$

One expects also this motivic cohomology to come endowed with Chern classes from algebraic $K$-theory. In fact, if one uses the decomposition $K_{n}(X) \otimes \mathbb{Q}=$ $\oplus_{j} K_{n}(X)^{(j)}$, where the Adams operation $\Psi_{k}$ acts on $K_{n}(X)^{(j)}$ as $k^{j}$, then one expects isomorphisms given by Chern classes

$$
c h^{j}: K_{n}(X)^{(j)} \stackrel{\simeq}{\rightrightarrows} H_{m o t}^{2 j-n}(X, \mathbb{Q}(j)) .
$$

Such motivic cohomology will be universal with respect to all cohomology theories for algebraic varieties satisfying certain natural properties (Bloch-Ogus axioms). Namely, for any such cohomology $H^{*}(\cdot, \Gamma(*))$ there will be a natural transformation $H_{m o t}^{*}(\cdot, \mathbb{Z}(*)) \rightarrow H^{*}(\cdot, \Gamma(*))$, compatible with the above isomorphisms. Mixed motives have increasing weight filtrations preserved by the realizations to cohomology theories. More generally, instead of working over a field $k$, one can consider a category $\mathcal{M}_{\text {mix }}(S)$ of motives (or "motivic sheaves") over a scheme $S$. In this case, the functors above are natural in $S$ and to a map of schemes $f: S_{1} \rightarrow S_{2}$ there correspond functors $f^{*}, f_{*}, f^{!}, f_{!}$, behaving like the corresponding functors of sheaves. 
The motivic Galois group for mixed motives will then be an extension of the proreductive motivic Galois group of pure motives by a pro-unipotent group. The prounipotent property reflects the presence of the weight filtration on mixed motives.

Though, at present, there is not yet a general construction of such a category of mixed motives $\mathcal{M}_{\text {mix }}(S)$, there are constructions of a triangulated tensor category $\mathcal{D} \mathcal{M}(S)$, which has the right properties to be the bounded derived category of the category of mixed motives. The constructions of $\mathcal{D} \mathcal{M}(S)$ due to Levine [46] and Voevodsky 61] are known to be equivalent. In general, given a construction of a triangulated tensor category, one can extract from it an abelian category by considering the heart of a t-structure. A caveat with this procedure is that it is not always the case that the given triangulated tensor category is in fact the bounded derived category of the heart of a $t$-structure. The available constructions, in any case, are obtained via this general procedure of $t$-structures developed in [3], which can be summarized as follows. A triangulated category $\mathcal{D}$ is an additive category with an automorphism $T$ and a family of distinguished triangles $X \rightarrow$ $Y \rightarrow Z \rightarrow T(X)$, satisfying suitable axioms (which we do not recall here). We use the notation $\mathcal{D}^{\geq n}=\mathcal{D}^{\geq 0}[-n]$ and $\mathcal{D}^{\leq n}=\mathcal{D}^{\leq 0}[-n]$, with $X[n]=T^{n}(X)$ and $f[n]=T^{n}(f)$. A $t$-structure consists of two full subcategories $\mathcal{D} \leq 0$ and $\mathcal{D}^{\geq 0}$ with the properties: $\mathcal{D}^{\leq-1} \subset \mathcal{D}^{\leq 0}$ and $\mathcal{D}^{\geq 1} \subset \mathcal{D}^{\geq 0}$; for all $X \in \mathcal{D}^{\leq 0}$ and all $Y \in \mathcal{D}^{\geq 1}$ one has $\operatorname{Hom}_{\mathcal{D}}(X, Y)=0$; for all $Y \in \mathcal{D}$ there exists a distinguished triangle as above with $X \in \mathcal{D}^{\leq 0}$ and $Z \in \mathcal{D}^{\geq 1}$. The heart of the t-structure is the full subcategory $\mathcal{D}^{0}=\mathcal{D}^{\leq 0} \cap \mathcal{D}^{\geq 0}$. It is an abelian category. This type of construction may be familiar to physicists in the context of mirror symmetry, where continuous families of hearts of $t$-structures play a role in [28.

For our purposes, we will be mostly interested in the full subcategory of Tate motives. The triangulated category of mixed Tate motives $\mathcal{D M T}(S)$ is then defined as the full triangulated subcategory of $\mathcal{D} \mathcal{M}(S)$ generated by the Tate objects. It is possible to define on it a $t$-structure whose heart gives a category of mixed Tate motives $\mathcal{M T}_{\text {mix }}(S)$, provided the Beilinson-Soulé vanishing conjecture holds, namely when

$$
\operatorname{Hom}^{j}(\mathbb{Q}(0), \mathbb{Q}(n))=0, \quad \text { for } n>0, j \leq 0 .
$$

where $\operatorname{Hom}^{j}(M, N)=\operatorname{Hom}(M, N[j])$. The conjecture (3.3) is known to hold in the case of a number field, where one has

$$
\operatorname{Ext}^{1}(\mathbb{Q}(0), \mathbb{Q}(n))=K_{2 n-1}(k) \otimes \mathbb{Q}
$$

and $\operatorname{Ext}^{2}(\mathbb{Q}(0), \mathbb{Q}(n))=0$. Thus, in this case it is possible to extract from the triangulated tensor category a Tannakian category $\mathcal{M T}_{\text {mix }}(k)$ of mixed Tate motives, with fiber functor $\omega$ to $\mathbb{Z}$-graded $\mathbb{Q}$-vector spaces, $M \mapsto \omega(M)=\oplus_{n} \omega_{n}(M)$ with

$$
\omega_{n}(M)=\operatorname{Hom}\left(\mathbb{Q}(n), \operatorname{Gr}_{-2 n}^{w}(M)\right),
$$

where $\operatorname{Gr}_{-2 n}^{w}(M)=W_{-2 n}(M) / W_{-2(n+1)}(M)$ is the graded structure associated to the finite increasing weight filtration $W$.

The motivic Galois group of the category $\mathcal{M T}_{\text {mix }}(k)$ is then an extension $G=$ $U \rtimes \mathbb{G}_{m}$, where the reductive piece is $\mathbb{G}_{m}$ as in the case of pure Tate motives, while $U$ is pro-unipotent. By the results of Goncharov (see [30, 24]), it is known that the pro-unipotent affine group scheme $U$ corresponds to a graded Lie algebra Lie $(U)$ that is free with one generator in each odd degree $n \leq-3$. 
A similar construction is possible in the case of the category $\mathcal{M} \mathcal{T}_{\text {mix }}(S)$, where the scheme $S$ is the set of $V$-integers $\varnothing_{V}$ of a number field $k$, for $V$ a set of finite places of $k$. In this case, objects of $\mathcal{M} \mathcal{T}_{\text {mix }}\left(\varnothing_{V}\right)$ are mixed Tate motives over $k$ that are unramified at each finite place $v \notin V$. For $\mathcal{M} \mathcal{T}_{\text {mix }}\left(\varnothing_{V}\right)$ we have

$$
\operatorname{Ext}^{1}(\mathbb{Q}(0), \mathbb{Q}(n))= \begin{cases}K_{2 n-1}(k) \otimes \mathbb{Q} & n \geq 2 \\ \emptyset_{V}^{*} \otimes \mathbb{Q} & n=1 \\ 0 & n \leq 0 .\end{cases}
$$

and $\operatorname{Ext}^{2}(\mathbb{Q}(0), \mathbb{Q}(n))=0$. In fact, the difference between the Ext in $\mathcal{M} \mathcal{T}_{\text {mix }}\left(\varnothing_{V}\right)$ of (3.6) and the Ext in $\mathcal{M} \mathcal{T}_{m i x}(k)$ of (3.4) is the $\operatorname{Ext}^{1}(\mathbb{Q}(0), \mathbb{Q}(1))$ which is finite dimensional in (3.6) and infinite dimensional in (3.4). The category $\mathcal{M T}_{\text {mix }}\left(\varnothing_{V}\right)$ is also a neutral Tannakian category, and the fiber functor determines an equivalence of categories between $\mathcal{M T}_{\text {mix }}\left(\varnothing_{V}\right)$ and finite dimensional linear representations of an affine group scheme of the form $U \rtimes \mathbb{G}_{m}$ with $U$ pro-unipotent. The Lie algebra $\operatorname{Lie}(U)$ is freely generated by a set of homogeneous generators in degree $n$ identified with a basis of the dual of $\operatorname{Ext}^{1}(\mathbb{Q}(0), \mathbb{Q}(n))$ (cf. Prop. 2.3 of 24$)$. There is however no canonical identification between $\operatorname{Lie}(U)$ and the free Lie algebra generated by the graded vector space $\oplus \operatorname{Ext}^{1}(\mathbb{Q}(0), \mathbb{Q}(n))^{\vee}$.

We mention the following case, which will be the one most relevant in the context of perturbative renormalization.

Proposition 3.1. (24, 30]) Consider the scheme $S_{N}=\varnothing[1 / N]$ for $k=\mathbb{Q}\left(\zeta_{N}\right)$ the cyclotomic field of level $N$ and $\varnothing$ its ring of integers. For $N=3$ or 4 , the motivic Galois group of the category $\mathcal{M T}_{\text {mix }}\left(S_{N}\right)$ is of the form $U \rtimes \mathbb{G}_{m}$, where the Lie algebra $\operatorname{Lie}(U)$ is (noncanonically) isomorphic to the free Lie algebra with one generator $e_{n}$ in each degree $n \leq-1$.

\section{Hilbert's XXI problem and the Riemann-Hilbert Correspondence}

Consider an algebraic linear ordinary differential equation, in the form of a system of rank $n$

$$
\frac{d}{d z} f(z)+A(z) f(z)=0
$$

on some open set $U=\mathbb{P}^{1}(\mathbb{C}) \backslash\left\{a_{1}, \ldots a_{r}\right\}$, where $A(z)$ is an $n \times n$ matrix of rational functions on $U$. In particular, this includes the case of a linear scalar $n$th order differential equation. The space $\mathcal{S}$ of germs of holomorphic solutions of (4.1) at a point $z_{0} \in U$ is an $n$-dimensional complex vector space. Moreover, given any element $\ell \in \pi_{1}\left(U, z_{0}\right)$, analytic continuation along a loop representing the homotopy class $\ell$ defines a linear automorphism of $\mathcal{S}$, which only depends on the homotopy class $\ell$. This defines the monodromy representation $\rho: \pi_{1}\left(U, z_{0}\right) \rightarrow \operatorname{Aut}(\mathcal{S})$ of the differential system (4.1). A slightly different formulation requires not the Fuchsian condition $(A(z)$ has simple poles) but the weaker regular singular condition for (4.1). The regularity condition at a singular point $a_{i} \in \mathbb{P}^{1}(\mathbb{C}) \backslash U$ is a growth condition on the solutions, namely all solutions in any strict angular sector centered at $a_{i}$ have at most polynomial growth in $1 /\left|z-a_{i}\right|$. The system (4.1) is regular singular if every $a_{i} \in \mathbb{P}^{1}(\mathbb{C}) \backslash U$ is a regular singular point. The Hilbert 21st problem (or Riemann-Hilbert problem) asks whether any finite dimensional complex linear representation of $\pi_{1}\left(U, z_{0}\right)$ is the monodromy representation of a differential system 
(4.1) with regular (or Fuchsian) singularities at the points of $\mathbb{P}^{1}(\mathbb{C}) \backslash U$. A solution to the Hilbert 21st problem in the regular singular case is given by Plemelj's theorem (cf. [1] §3). The argument first produces a system with the assigned monodromy on $U$, where in principle an analytic solution has no constraint on the behavior at the singularities. Then, one restricts to a local problem in small punctured disks $\Delta^{*}$ around each of the singularities, for which a system exists with the prescribed behavior of solutions at the origin. The global trivialization of the holomorphic bundle on $U$ determined by the monodromy datum yields the patching of these local problems that produces a global solution with the correct growth condition at the singularities.

\section{From problem to correspondence.}

A modern revival of interest in Fuchsian differential equation, with a new algebraic viewpoint that slowly transformed the original Riemann-Hilbert problem into the broad landscape of the Riemann-Hilbert correspondence, was pioneered in the early 1960s by the influential paper of Yuri Manin [47] on Fuchsian modules. This new perspective influenced the work of Deligne 21] in 1970, who solved the RiemannHilbert problem for regular singular equations on an arbitrary smooth projective variety. In this viewpoint, if $X$ is a smooth projective variety and $U$ is a Zariski open set, with $X \backslash U$ a union of divisors with normal crossing, the data of an algebraic differential system are given by a pair $(M, \nabla)$ of a locally free coherent sheaf on $U$ with a connection $\nabla: M \rightarrow M \otimes \Omega_{U / \mathbb{C}}^{1}$, while the regular singular condition says that there exists an algebraic extension $(\bar{M}, \bar{\nabla})$ of the data $(M, \nabla)$ to $X$, where the extended connection $\bar{\nabla}: \bar{M} \rightarrow \bar{M} \otimes \Omega_{X / \mathbb{C}}^{1}(\log D)$ has $\log$ singularities at the divisor $D$. The reconstruction argument for algebraic linear differential systems with regular singularities in terms of their monodromy representation consists then of first producing an analytic solution $(M, \nabla)$ on $U$ with the prescribed monodromy and then restricting to a local problem in punctured polydisks $\Delta^{*}$ around the singularities, to obtain a local extension of the form $H(z) \prod_{j} z_{j}^{B_{j}}$, where $H \in$ $\mathrm{GL}_{n}\left(\varnothing_{\Delta^{*}}\right)$ and the $B_{j}$ are commuting matrices that give the local monodromy representation $\exp \left(2 \pi i B_{j}\right)$ of $\pi_{1}\left(\Delta^{*}\right)$. An important point of the argument is to show that these local extensions can be patched together. The patching problem does not arise when $\operatorname{dim} U=1$, since in that case the divisor $D$ consists of isolated points. The construction is then completed by showing that the global analytic extension $(\bar{M}, \bar{\nabla})$ obtained this way on $X$ is equivalent to an algebraic extension.

Starting with the early 1980s, with the work of Mebkhout [51] [52] and of Kashiwara 38 39, and with the development of the theory of perverse sheaves by Beilinson, Bernstein, Deligne, and Gabber [3], the Riemann-Hilbert correspondence was recast in terms of an equivalence of derived categories between regular holonomic $\mathcal{D}_{X^{-}}$ module and perverse sheaves. A reason for introducing the language of $\mathcal{D}$-modules (cf. e.g. 29] $\S 8$ or [45] for an overview) is that this captures more information in a differential system $(M, \nabla)$, than what was possible with the previous formulations. For instance, the data $(M, \nabla)$ fit into a de Rham type complex. Also, one may want to consider different classes of solutions (smooth, holomorphic, distributional, etc). This type of extra information is taken care of by the formalism of $\mathcal{D}$-modules. Namely, a differential equation determines a module $\mathcal{M}$ over $\mathcal{D}_{X}$ (differential operators on $X$ with holomorphic coefficients), with solutions to the equation given by $\operatorname{Hom}_{\mathcal{D}_{X}}\left(\mathcal{M}, \mathcal{O}_{X}\right)$. One can alter the type of solutions by replacing $\mathcal{O}_{X}$ by another 
module $\mathcal{N}$ over $\mathcal{D}_{X}$, and account for the extra structure in the data $(M, \nabla)$ by considering the de Rham complex $\mathcal{M} \otimes_{\mathcal{O}_{X}} \Omega_{X}$. The condition of regular singularities can be extended to modules $\mathcal{M}$ subject to another 'growth' condition, related to the module structure, compatibly with a natural filtration of $\mathcal{D}_{X}$ (holonomic $\mathcal{D}$ modules). Then the equivalence of categories extends to an equivalence of derived categories, between regular holonomic $\mathcal{D}_{X}$-module and perverse sheaves.

With the regular singular hypothesis replaced by the stronger Fuchsian condition, as in Hilbert's original formulation, counterexamples to the Riemann-Hilbert problem were later found by Bolibruch $\left[8\right.$, in the simplest case of $X=\mathbb{P}^{1}(\mathbb{C})$. On the other hand, one can instead relax the regular singular condition and look at classes of differential systems with irregular singularities. It is immediately clear that finite dimensional complex linear representations of the fundamental group no longer suffice to distinguish equations that can have very different analytic behavior at the singularities and equal monodromy. One can see this in a simple example, where all equations of the form $\frac{d}{d z} f(z)+\frac{1}{z^{2}} P\left(\frac{1}{z}\right) f(z)=0$ have trivial monodromy, for any polynomial $P$, but they all have inequivalent behavior at the singularity $z=0$.

Thus, one needs a refinement of the fundamental group, whose finite dimensional linear representations are equivalent to (a given class of) irregular differential systems. There are different approaches to the irregular case. Since we are directly interested in the case relevant to perturbative renormalization, we might as well restrict our attention to the one dimensional setting, namely where $\operatorname{dim} U=1$ and $X$ is a compact Riemann surface. In fact, in our case $X=\mathbb{P}^{1}(\mathbb{C})$ will be sufficient, as we will be interested only in the local problem in a punctured disk $\Delta^{*}$. As we discuss in Section 5 below, in physical terms $\Delta^{*}$ represents the space of complexified dimensions around a given integer dimension $D$ at which the Feynman integrals of the specified theory $\mathcal{T}$ are divergent.

In this context, the theory that best fits our needs for the application to renormalization was developed by Martinet and Ramis [50, where instead of the usual fundamental group one considers representations of a wild fundamental group, which arises from the asymptotic theory of divergent series and differential Galois theory.

\section{Differential Galois theory and the wild fundamental group.}

We consider a local version of the irregular Riemann-Hilbert correspondence, in a small punctured disk $\Delta^{*}$ in the complex plane around a singularity $z=0$. We work in the context of differential Galois theory $(c f$. [53, [54]). In this setting, one works over a differential field $(K, \delta)$, such that the field of constants $k=\operatorname{Ker}(\delta)$ is an algebraically closed field of characteristic zero. One considers differential systems of the form $\delta f=A f$, for some $A \in \operatorname{End}(n, K)$.

For $k=\mathbb{C}$, at the formal level we are then working over the differential field of formal complex Laurent series $K=\mathbb{C}((z))=\mathbb{C}[[z]]\left[z^{-1}\right]$, with differentiation $\delta=z \frac{d}{d z}$, while at the non-formal level one considers the subfield $K=\mathbb{C}(\{z\})$ of convergent Laurent series.

Given a differential system $\delta f=A f$, its Picard-Vessiot ring is a $K$-algebra with a differentiation extending $\delta$. As a differential algebra it is simple and is generated over $K$ by the entries and the inverse determinant of a fundamental matrix for the 
equation $\delta f=A f$. The differential Galois group of the differential system is given by the automorphisms of the Picard-Vessiot ring commuting with $\delta$.

The formalism of Tannakian categories, that we discussed in Section 3 in the context of motives, reappears in the present context and allows for a description of the differential Galois group that fits in the same general picture we recalled regarding motivic Galois groups.

In fact, if we consider the set of all possible such differential systems (differential modules over $K$ ), these form a neutral Tannakian category, which can therefore be identified with the category of finite dimensional linear representations of a unique affine group scheme over the field $k$.

Similarly to what we discussed in the case of motivic Galois groups, any subcategory $\mathbb{T}$ that inherits the structure of a neutral Tannakian category in turn corresponds to an affine group scheme $G_{\mathbb{T}}$. This is the universal differential Galois group of the class of differential systems that form the category $\mathbb{T}$. It can be realized as the automorphisms group of the universal Picard-Vessiot ring $R_{\mathbb{T}}$. The latter is generated over $K$ by the entries and inverse determinants of the fundamental matrices of all the differential systems considered in the category $\mathbb{T}$.

There is therefore a clear analogy between the induced motivic Galois groups of certain subcategories of, say, the category of mixed Tate motives that we discussed in Section 3 and the differential Galois group of certain classes of differential systems defining subcategories of the neutral Tannakian category of irregular differential systems over a differential field $K$. Our main result of [18, which we discuss in Section 5 below, shows that the theory of perturbative renormalization (in the DimReg and minimal subtraction scheme) identifies a class of differential systems (dictated by physical assumptions), whose differential Galois group is the motivic Galois group of Proposition 3.1

The regular-singular case can be seen in this context as follows. The subcategory of regular-singular differential modules over $K=\mathbb{C}((z))$ is a neutral Tannakian category equivalent to $\operatorname{Rep}_{G}$, where the affine group scheme $G$ is the algebraic hull $\overline{\mathbb{Z}}$ of $\mathbb{Z}$, generated by the formal monodromy $\gamma$. The latter is the automorphism of the universal Picard-Vessiot ring acting by $\gamma Z^{a}=\exp (2 \pi i a) Z^{a}$ and $\gamma L=L+2 \pi i$ on the generators $\left\{Z^{a}\right\}_{a \in \mathbb{C}}$ and $L$, which correspond, in angular sectors, to the powers $z^{a}$ and the function $\log (z)$ (cf. [53] §III, [54]).

When one allows for an arbitrary degree of irregularity for the differential systems $\delta f=A f$, the universal Picard-Vessiot ring of the formal theory $K=\mathbb{C}((z))$ is generated by elements $\left\{Z^{a}\right\}_{a \in \mathbb{C}}$ and $L$ as before, and by additional elements $\{E(q)\}_{q \in \mathcal{E}}$, where $\mathcal{E}=\cup_{\nu \in \mathbb{N} \times} \mathcal{E}_{\nu}$, for $\mathcal{E}_{\nu}=z^{-1 / \nu} \mathbb{C}\left[z^{-1 / \nu}\right]$. These additional generators correspond, in local sectors, to functions of the form $\exp \left(\int q \frac{d z}{z}\right)$ and satisfy relations $E\left(q_{1}+q_{2}\right)=E\left(q_{1}\right) E\left(q_{2}\right)$ and $\delta E(q)=q E(q)$.

Correspondingly, the universal differential Galois group $\mathcal{G}$ is described by an extension $\mathcal{T} \rtimes \overline{\mathbb{Z}}$, where $\mathcal{T}=\operatorname{Hom}\left(\mathcal{E}, \mathbb{C}^{*}\right)$ is the Ramis exponential torus. The algebraic hull $\overline{\mathbb{Z}}$ generated by the formal monodromy $\gamma$ acts as an automorphism of the universal Picard-Vessiot ring by the same action as above on the $Z^{a}$ and on $L$, and by $\gamma E(q)=E(\gamma q)$ on the additional generators, where the action on $\mathcal{E}$ is given by the action $\gamma: q\left(z^{-1 / \nu}\right) \mapsto q\left(\exp (-2 \pi i / \nu) z^{-1 / \nu}\right)$ of $\mathbb{Z} / \nu \mathbb{Z}$ on $\mathcal{E}_{\nu}$. The exponential torus acts by automorphisms of the universal Picard-Vessiot $\operatorname{ring} \tau Z^{a}=Z^{a}, \tau L=L$ 
and $\tau E(q)=\tau(q) E(q)$, and the formal monodromy acts on the exponential torus by $(\gamma \tau)(q)=\tau(\gamma q)$.

Thus, at the formal level, the local irregular Riemann-Hilbert correspondence establishes an equivalence of categories between the differential modules over $K=\mathbb{C}((z))$ and finite dimensional linear representations of $G=\mathcal{T} \rtimes \overline{\mathbb{Z}}$. Ramis' wild fundamental group [50] further extends this irregular Riemann-Hilbert correspondence to the non-formal setting. In general, when passing to the non-formal level over convergent Laurent series $K=\mathbb{C}(\{z\})$, the universal differential Galois group acquires additional generators, which depend upon resummation of divergent series and are related to the Stokes phenomenon. However, there are specific classes of differential systems (subcategories of differential modules over $K$ ), for which the differential Galois group is the same over $\mathbb{C}((z))$ and over $\mathbb{C}(\{z\})$ (cf. e.g. Proposition 3.40 of [53]). In such cases, the wild fundamental group consists only of the exponential torus and the formal monodromy. This is, in fact, the case in the class of differential systems we obtain from the theory of perturbative renormalization, hence we do not need to discuss here the more complicated case where Stokes phenomena are present, and we simply refer the interested reader to [50], [53], and [54].

\section{Cartier's dream of a cosmic Galois group}

In the section "I have a dream" of [9], Pierre Cartier formulated the hypothesis of the existence of a "cosmic Galois group", closely related to the GrothendieckTeichmüller group [32, underlying the Connes-Kreimer theory of perturbative renormalization, that would relate quantum field theory to the theory of motives and multiple zeta values.

We present in this section the main result of [18, which realizes Cartier's suggestion, by reformulating the Connes-Kreimer theory of perturbative renormalization in the form of a suitable Riemann-Hilbert correspondence.

\section{Equisingular connections.}

The first step, in order to pass to this type of geometric formulation, is to identify the loops $\gamma_{\mu}(z)=\gamma_{\mu,-}(z)^{-1} \gamma_{\mu,+}(z)$ with solutions of suitable differential equations. The idea of reformulating a Birkhoff factorization problem in terms of a class of differential equations is familiar to the analytic approach to the Riemann-Hilbert problem ( $c f$. [7]). In our setting, the key that allows us to pass from the Birkhoff factorization to an appropriate class of differential systems is provided by the 't Hooft relations in the form of Proposition 2.2 and the scattering formula (2.13), reformulated more explicitly in terms of iterated integrals.

Here the main tool is the time ordered exponential, formulated mathematically in terms of Chen's iterated integrals 11], 12, also known (in the operator algebra context) as Araki's expansional [2].

We consider a commutative Hopf algebra $\mathcal{H}$ that is graded in positive degree and connected, with $G$ the corresponding affine group scheme and $\mathfrak{g}=$ Lie $G$. We assume that $\mathcal{H}$ is, in each degree, a finite dimensional vector space. Given a $\mathfrak{g}(\mathbb{C})$-valued smooth function $\alpha(t)$ where $t \in[a, b] \subset \mathbb{R}$ is a real parameter, the expansional is defined by the expression

$$
\mathrm{Te}^{\int_{\mathbf{a}}^{\mathbf{b}} \alpha(\mathbf{t}) \mathbf{d} \mathbf{t}}=1+\sum_{1}^{\infty} \int_{a \leq s_{1} \leq \cdots \leq s_{n} \leq b} \alpha\left(s_{1}\right) \cdots \alpha\left(s_{n}\right) \prod d s_{j},
$$


where the products are in the dual algebra $\mathcal{H}^{*}$ and $1 \in \mathcal{H}^{*}$ is the unit given by the augmentation $\varepsilon$. When paired with any element $x \in \mathcal{H}$, (5.1) reduces to a finite sum, which defines an element in $G(\mathbb{C})$.

The fact that, when pairing with elements in $\mathcal{H}$ one reduces to an algebraic (polynomial) case plays an important role. In particular, it is related to the fact that, for the class of differential systems we consider, the differential Galois group remains the same in the formal and in the non-formal case, and we need not take into account the possible presence of Stokes' phenomena.

We are particularly interested in the following property of the expansional: (5.1) is the value $g(b)$ at $b$ of the unique solution $g(t) \in G(\mathbb{C})$ with value $g(a)=1$ of the differential equation

$$
d g(t)=g(t) \alpha(t) d t .
$$

More generally, if $(K, \delta)$ is a differential field with $K \supset \mathbb{C}$ and if $g \in G(K)$ and $g^{\prime}=\delta(g)$ is the linear map $\mathcal{H} \rightarrow K$ defined as $g^{\prime}(x)=\delta(g(x))$ for $x \in \mathcal{H}$, then the logarithmic derivative $D(g)$ is defined as the linear map $\mathcal{H} \rightarrow K$ of the form $D(g)=g^{-1} \star g^{\prime}$, with the product dual to the coproduct of $\mathcal{H}$. It satisfies

$$
\langle D(g), x y\rangle=\langle D(g), x\rangle \varepsilon(y)+\varepsilon(x)\langle D(g), y\rangle, \quad \forall x, y \in \mathcal{H},
$$

hence it gives an element in the Lie algebra $D(g) \in \mathfrak{g}(K)$. We will work here with the field of convergent Laurent series $K=\mathbb{C}(\{z\})$.

If we consider over $\Delta^{*}$ a differential system of the form

$$
D f=\omega,
$$

where $\omega$ is a flat $\mathfrak{g}(K)$-valued connection, then the condition of trivial monodromy

$$
\mathrm{Te}^{\int_{\mathbf{0}}^{1} \gamma^{*} \omega}=1,
$$

for $\gamma \in \pi_{1}\left(\Delta^{*}, z_{0}\right)$, ensures the existence of a solution. In the expansional form this is given by

$$
g(z)=\mathrm{Te}^{\int_{\mathbf{z}_{0}}^{\mathbf{z}} \omega},
$$

independently of the path in $\Delta^{*}$ from $z_{0}$ to $z$.

The notion of equivalence relation that we consider for differential systems of the form (5.3) is the following: two connections $\omega$ and $\omega^{\prime}$ are equivalent iff they are related by a gauge transformation $h \in G(\varnothing)$, with $\varnothing \subset K$ the subalgebra of regular functions,

$$
\omega^{\prime}=D h+h^{-1} \omega h
$$

The behavior of solutions at the singularity is the same for all equivalent connections. When we regard the solutions as $G(\mathbb{C})$-valued loops, the equivalence (5.6) of the connections translates to the fact that the loops have the same negative part of the Birkhoff decomposition.

So far we have not taken into account the fact that, in the case of perturbative renormalization, the loop $\gamma_{\mu}(z)$ that corresponds to the unrenormalized theory depends on the mass parameter $\mu$, as discussed above in Section 2 Because of the presence of this parameter, the geometric reformulation in terms of a class of differential systems takes place, in fact, not just on the 1-dimensional (infinitesimal) punctured disk $\Delta^{*}$ representing the complexified dimensions of DimReg, but on a principal $\mathbb{G}_{m}(\mathbb{C})=\mathbb{C}^{*}$-bundle over $\Delta^{*}$. 
As we discuss below, the fact that the loop $\gamma_{\mu}(z)$ satisfies the properties (2.5) and (2.6) will make it possible to treat this case, which lives naturally over a 2dimensional space, by applying the same techniques described in Section 4 for the irregular Riemann-Hilbert correspondence over the 1-dimensional domain $\Delta^{*}$.

The conditions (2.5) and (2.6) determine a class of differential systems associated to perturbative renormalization. This is given by equivalence classes of flat equisingular $G(\mathbb{C})$-connections, where $G=\operatorname{Difg}(\mathcal{T})$.

Let $\pi: B \rightarrow \Delta$ be a principal $\mathbb{G}_{m}(\mathbb{C})=\mathbb{C}^{*}$-bundle, identified with $\Delta \times \mathbb{C}^{*}$ by the non-canonical choice of a section $\sigma: \Delta \rightarrow B, \sigma(0)=y_{0}$. Physically, the latter corresponds to a choice of the Planck constant. Let $P=B \times G(\mathbb{C})$ be the trival principal $G(\mathbb{C})$-bundle, and $B^{*}$ and $P^{*}$ the restrictions to the punctured disk $\Delta^{*}$.

We say that the connection $\omega$ on $P^{*}$ is equisingular if it is $\mathbb{G}_{m}$-invariant and its restrictions to sections of the principal bundle $B$ that agree at $0 \in \Delta$ are mutually equivalent, in the sense that they are related by a gauge transformation by a $G(\mathbb{C})$ valued $\mathbb{G}_{m}$-invariant map $h$ regular in $B$.

The notion of equisingularity is introduced as a geometric reformulation of the properties (2.5) and (2.6). In fact, the property that, when approaching the singular fiber, the type of singularity does not depend on the section along which one restricts the connection but only on the value of the section at $0 \in \Delta$ corresponds to the fact that the counterterms are independent of the mass scale, as in (2.5).

Thus, we have identified a class of differential systems associated to a physical theory $\mathcal{T}$, namely the equivalence classes of flat equisingular $G(\mathbb{C})$-valued connections on $P$, where $G=\operatorname{Difg}(\mathcal{T})$. We can then proceed to investigate the Riemann-Hilbert correspondence underlying this class of differential systems.

The first step consists of writing solutions of (2.5) and (2.6) in expansional form through the following result, which we can view as a stronger version of the ' $t$ Hooft relations.

Proposition 5.1. Let $\gamma_{\mu}(z)$ be a family of $G(\mathbb{C})$-valued loops satisfying the properties (2.5) and (2.6). Then there exists a unique $\beta \in \operatorname{Lie} G(\mathbb{C})$ and a loop $\gamma_{\mathrm{reg}}(z)$ regular at $z=0$, such that

$$
\gamma_{\mu}(z)=\mathrm{T} \mathbf{e}^{-\frac{1}{\mathbf{z}} \int_{\infty}^{-\mathbf{z} \log \mu} \theta_{-\mathbf{t}}(\beta) \mathbf{d t}} \theta_{z \log \mu}\left(\gamma_{\mathrm{reg}}(z)\right) .
$$

Conversely, for any $\beta$ and regular loop $\gamma_{\mathrm{reg}}(z)$ the expression (5.7) gives a solution to (2.5) and (2.6). The Birkhoff decomposition of $\gamma_{\mu}(z)$ is of the form

$$
\begin{aligned}
& \gamma_{\mu^{+}}(z)=\mathrm{Te}^{-\frac{1}{\mathbf{z}} \int_{\mathbf{0}}^{-\mathbf{z} \log \mu} \theta_{-\mathbf{t}}(\beta) \mathbf{d t}} \theta_{z \log \mu}\left(\gamma_{\mathrm{reg}}(z)\right), \\
& \gamma_{-}(z)=\mathrm{T}^{-\frac{1}{z} \int_{0}^{\infty} \theta_{-\mathbf{t}}(\beta) \mathbf{d t}} .
\end{aligned}
$$

Using the equivalent geometric formulation in terms of flat equisingular connections, one then obtains the following result.

Proposition 5.2. Let $\omega$ be a flat equisingular $G(\mathbb{C})$-connection. There exists a unique element $\beta \in \operatorname{Lie} G(\mathbb{C})$, such that $\omega$ is equivalent to the flat equisingular connection $D \gamma$ for

$$
\gamma(z, v)=\mathrm{Te}^{-\frac{1}{\mathbf{z}} \int_{\mathbf{0}}^{\mathbf{v}} \mathbf{u}^{\mathbf{Y}}(\beta) \frac{\mathrm{du}}{\mathbf{u}}} \in G(\mathbb{C}),
$$

with the integral performed on the straight path $u=t v, t \in[0,1]$. 
Here a crucial point is the fact that the monodromies with respect to the two generators of $\pi_{1}\left(B^{*}\right)$ vanish for flat equisingular connections. As we will see in the next section, this fact will be reflected in the form of the affine group scheme associated to the category of equivalence classes of flat equisingular connections (the differential Galois group), which will only contain the part corresponding to the Ramis exponential torus and no contribution from the monodromy. The correspondence of Proposition 5.2 is independent of the choice of the trivialising section $\sigma$ of $B$.

\section{The Riemann-Hilbert correspondence.}

So far we have been working with an assigned quantum field theory $\mathcal{T}$ and the corresponding affine group scheme $G=\operatorname{Difg}(\mathcal{T})$. We now pass to considering a universal setting, which encompasses all theories. This is achieved by considering, instead of flat equisingular $G(\mathbb{C})$-connections, the category of equivalence classes of all flat equisingular bundles. For a specific physical theory, the corresponding category of equivalence classes of flat equisingular $G(\mathbb{C})$-connections can be recovered from this more general setting by considering the subcategory of those flat equisingular bundles that are finite dimensional linear representations of $G^{*}=G \rtimes \mathbb{G}_{m}$. This is analogous to what happens when one specializes motivic Galois groups to sucategories of motives, or differential Galois groups to subcategories of differential systems. We describe now in detail the universal setting, with the corresponding group of symmetries and the way it specializes to a given physical theory.

The category of equivalence classes of flat equisingular bundles has as objects $\Theta=$ $(E, \nabla)$ pairs of a finite dimensional $\mathbb{Z}$-graded vector space $E$ and an equisingular flat $W$-connection $\nabla$. To define the latter, we consider the vector bundle $\tilde{E}=B \times E$ with the action of $\mathbb{G}_{m}$ given by the grading and with the weight filtration defined by $W^{-n}(E)=\oplus_{m \geq n} E_{m}$. A $W$-connection is a connection on the restriction of $\tilde{E}$ to $B^{*}$, which is compatible with the weight filtration and induces the trivial connection on the associated graded. The connection $\nabla$ in the data above is a flat $W$-connection that satisfies the equisingular condition, that is, it is $\mathbb{G}_{m}$-invariant and the restrictions to sections $\sigma$ of $B$ with $\sigma(0)=y_{0}$ are all $W$-equivalent on $B$, where the equivalence relation is realized by an isomorphism of the vector bundles over $B$, compatible with the filtration and identity on the associated graded, that conjugates the connections. We consider the data $\Theta=(E, \nabla)$ as $W$-equivalence classes.

As usual, it is a bit more delicate to define morphisms than objects.

For a linear map $T: E \rightarrow E^{\prime}$, consider the $W$-connections $\nabla_{j}, j=1,2$, on $\tilde{E}^{\prime} \oplus \tilde{E}$ of the form

$$
\nabla_{1}=\left(\begin{array}{cc}
\nabla^{\prime} & 0 \\
0 & \nabla
\end{array}\right) \quad \text { and } \quad \nabla_{2}=\left(\begin{array}{cc}
\nabla^{\prime} & T \nabla-\nabla^{\prime} T \\
0 & \nabla
\end{array}\right)
$$

where $\nabla_{2}$ is the conjugate of $\nabla_{1}$ by the unipotent matrix

$$
\left(\begin{array}{ll}
1 & T \\
0 & 1
\end{array}\right)
$$

Morphisms $T \in \operatorname{Hom}\left(\Theta, \Theta^{\prime}\right)$ in the category of equisingular flat bundles are linear maps $T: E \rightarrow E^{\prime}$ compatible with the grading and such that the connections $\nabla_{j}$ of (5.10) are $W$-equivalent on $B$. The condition is independent of the choice of representatives for the connections $\nabla$ and $\nabla^{\prime}$. 
The category $\mathcal{E}$ of equisingular flat bundles is a tensor category over $k=\mathbb{C}$, with a fiber functor $\omega: \mathcal{E} \rightarrow V e c t_{\mathbb{C}}$ given by

$$
\omega: \Theta=(E, \nabla) \mapsto E .
$$

In fact, one can refine the construction and work over the field $k=\mathbb{Q}$, since the universal singular frame (see (5.18) below), in which one expresses the connections, has rational coefficients. In this case, the fiber functor $\omega: \mathcal{E}_{\mathbb{Q}} \rightarrow V$ ect $\mathbb{Q}$ is of the form $\omega=\oplus \omega_{n}$, with

$$
\omega_{n}(\Theta)=\operatorname{Hom}\left(\mathbb{Q}(n), \operatorname{Gr}_{-n}^{W}(\Theta)\right),
$$

where $\mathbb{Q}(n)$ denotes the object in $\mathcal{E}_{\mathbb{Q}}$ given by the class of the pair of the trivial bundle over $B$ with fiber a one-dimensional $\mathbb{Q}$-vector space placed in degree $n$ and the trivial connection.

Let $\mathcal{F}(1,2,3, \cdots)$. be the free graded Lie algebra generated by one element $e_{-n}$ in each degree $n \in \mathbb{Z}_{>0}$, and let

$$
\mathcal{H}_{u}=\mathcal{U}(\mathcal{F}(1,2,3, \cdots) \bullet)^{\vee}
$$

be the commutative Hopf algebra obtained by considering the graded dual of the enveloping algebra $\mathcal{U}(\mathcal{F})$. We can then identify explicitly the affine group scheme associated to the neutral Tannakian category of flat equisingular bundles as follows (cf. [18] [19]).

Theorem 5.3. The category $\mathcal{E}$ of flat equisingular bundles is a neutral Tannakian category, with fiber functor (5.11). It is equivalent to the category $\operatorname{Rep}_{U^{*}}$ of finite dimensional linear representations of the affine group scheme $U^{*}=U \rtimes \mathbb{G}_{m}$, where $U$ is the pro-unipotent affine group scheme associated to the Hopf algebra $\mathcal{H}_{u}$ of (5.12).

The affine group scheme $U^{*}$ is a motivic Galois group. In fact, by results of Goncharov and Deligne ([24, [30], see Proposition 3.1] above), we have the following identification of the "cosmic Galois group" $U^{*}$.

Proposition 5.4. There is a (non-canonical) isomorphism

$$
U^{*} \cong G_{\mathcal{M}_{T}}(\mathcal{O}) \text {. }
$$

of the affine group scheme $U^{*}$ with the motivic Galois group $G_{\mathcal{M}_{T}}(\mathcal{O})$ of the scheme $S_{N}$ of $N$-cyclotomic integers, for $N=3$ or $N=4$.

The fact that we only have a noncanonical identification suggests that there should be an explicit identification dictated by the form of the iterated integrals that give the expansionals defining the equisingular connections as in Proposition 5.1. This should be related to Kontsevich's formula for multiple zeta values as iterated integrals generalized by Goncharov to multiple polylogarithms $\mathrm{Li}_{k_{1}, \ldots, k_{m}}\left(z_{1}, z_{2}, \ldots, z_{m}\right)$, in terms of the expansional $\mathrm{Te}^{\int_{\mathbf{0}}^{1} \alpha(\mathbf{z}) \mathbf{d z}}$, with the connection

$$
\alpha(z) d z=\sum_{a \in \mu_{m} \cup\{0\}} \frac{d z}{z-a} e_{a} .
$$

Notice, moreover, that the group $U^{*}$, as the differential Galois group in the formal theory of equisingular connections, corresponds to the Ramis exponential torus. In fact, we have no contribution from the monodromy, a fact on which the proof of Proposition 5.2 depends essentially, and we also do not have Stokes phenomena, 
hence, as far as the differential Galois group is concerned, we can equally work in the formal or in the non-formal setting.

\section{The renormalization group as a Galois group.}

The formulation of Theorem 5.3 is universal with respect to physical theories. When we consider a particular choice of a renormalizable theory $\mathcal{T}$, we restrict the category of equisingular flat bundles to the full subcategory of finite dimensional linear representations of $G^{*}=G \rtimes \mathbb{G}_{m}$, for $G=\operatorname{Difg}(\mathcal{T})$. In this case, the RiemannHilbert correspondence specializes to a morphism of differential Galois groups, as follows.

Proposition 5.5. Let $G$ be a positively graded pro-unipotent affine group scheme. Then there exists a canonical bijection between equivalence classes of flat equisingular $G(\mathbb{C})$-connections and graded representations $\rho: U \rightarrow G$, of the affine group scheme $U$ in $G$. Compatibility with the grading implies that $\rho$ extends to a homomorphism $\rho^{*}: U^{*} \rightarrow G^{*}$, which is the identity on $\mathbb{G}_{m}$.

This is a reformulation of the result of Proposition 5.2. In fact, more explicitly, the representation $\rho$ of Proposition [5.5] is obtained as follows. We can write an element $\beta$ in Lie $G$ as an infinite formal sum

$$
\beta=\sum_{1}^{\infty} \beta_{n}
$$

where, for each $n, \beta_{n}$ is homogeneous of degree $n$ for the grading, i.e. $Y\left(\beta_{n}\right)=n \beta_{n}$. Thus, assigning $\beta$ with the action of the grading is the same as giving a collection of homogeneous elements $\beta_{n}$, with no restriction besides $Y\left(\beta_{n}\right)=n \beta_{n}$. In particular, there is no condition on their Lie brackets, hence assigning such data is equivalent to giving a homomorphism from the affine group scheme $U$ to $G$, by assigning, at the Lie algebra level, the generator $e_{-n}$ to the component $\beta_{n}$ of $\beta$.

In particular, the result above means that we can realize the renormalization group as a Galois group. In fact, recall that, for an assigned theory $\mathcal{T}$, the corresponding $\beta$ that determines the counterterms $\gamma_{-}(z)$ is the infinitesimal generator of the renormalization group (2.9). The representation $\rho: U^{*} \rightarrow G^{*}$ then determines a lifting of the renormalization group rg to a canonical 1-parameter subgroup of $U^{*}$, obtained by considering the element

$$
e=\sum_{1}^{\infty} e_{-n},
$$

in the Lie algebra $\operatorname{Lie} U$. As $U$ is a pro-unipotent affine group scheme, $e$ defines a morphism of affine group schemes

$$
\text { rg: } \mathbb{G}_{a} \rightarrow U,
$$

from the additive group $\mathbb{G}_{a}$ to $U$.

Thus, the rest of the affine group scheme $U$ can be throught of as further symmetries that refine the action of the renormalization group on a given physical theory. More precisely, restricting the attention to a generator $e_{-n}$ of the Lie algebra of $U$ corresponds to considering the flow generated by the degree $n$ component of the $\beta$ function with respect to the grading by loop number. Thus, from a physical 
point of view the Galois group $U$ accounts for a decomposition of the action of the renormalization group in terms of a family of flows restricted to the $n$-loops theory.

\section{Universal singular frame.}

The element $e \in \operatorname{Lie} U$ defined in (5.15) determines a "universal singular frame" given by

$$
\gamma_{U}(z, v)=\mathrm{Te}^{-\frac{1}{z} \int_{\mathbf{0}}^{\mathbf{v}} \mathbf{u}^{\mathbf{Y}}(\mathbf{e}) \frac{\mathrm{du}}{\mathbf{u}}} \in U .
$$

This is obtained by applying Proposition 5.2 to the affine group scheme $U$. This can be expressed explicitly in terms of iterated integrals in the form

$$
\gamma_{U}(z, v)=\sum_{n \geq 0} \sum_{k_{j}>0} \frac{e_{-k_{1}} e_{-k_{2}} \cdots e_{-k_{n}}}{k_{1}\left(k_{1}+k_{2}\right) \cdots\left(k_{1}+k_{2}+\cdots+k_{n}\right)} v^{\sum k_{j}} z^{-n},
$$

with $e_{-n}$ the generators of Lie $U$. This expansion has rational coefficients. The coefficients are the same as those occurring in in the local index formula of ConnesMoscovici 20, where the renormalization group idea is used in the case of higher poles in the dimension spectrum.

The Birkhoff factorization in $U$, applied to the universal singular frame, yields universal counterterms that maps under the representation $\rho: U \rightarrow \operatorname{Difg}(\mathcal{T})$ to the counterterms of a specific theory $\mathcal{T}$.

\section{RenORMALizATION AND GEOMETRY}

Quantum mechanics allows for two equivalent formulations of physics at the macroscopic scale, based on coordinate and momentum space, dual to one another by Fourier transform, while gravity, relativistically formulated in terms of the geometry of space-time, appears to privilege coordinates over momenta.

In the quantum theory of fields, at the perturbative level, Feynman integrals are computed in momentum space, using the dimensional regularization scheme. A nice historical and motivational perspective on how this came to be the general "accepted paradigm" in the context of renormalizable perturbation theory can be found in Veltman's paper [60. As Veltman suggests, one can assume perturbative field theory as the starting point, defined in terms of Feynman diagrams using dimensional regularization (he refers to this as the "dimensional formulation"). This is very much the approach followed by the Connes-Kreimer theory and by our present work, where such physical data, taken as the given starting point, are reformulated in a more satisfactory conceptual perspective.

It is also possible to follow a different approach and to consider the problem of perturbative renormalization in coordinate space, working geometrically in terms of Fulton-MacPherson compactifications. A mathematical theory of perturbative renormalization under this point of view was developed recently by Kontsevich 41]. It has the advantage of introducing directly geometric objects like algebraic varieties, hence a natural setting for an explicit action of motivic Galois symmetries (cf. also [40]).

As stressed by Veltman [60, space and time do not occur at all in the dimensional formulation, as coordinate space exists solely as Fourier transform of momentum space, which ceases to be defined when momentum space is continued to complex dimension. Notions associated to coordinate space, such as length and time measurements, must be recovered through the gravitational field, with graviton-fermion 
interactions determined by gauge invariance and Ward identities. Thus, a viewpoint that favors momentum rather than coordinate space is necessarily closer to noncommutative geometry than to classical algebraic geometry. In noncommutative geometry the metric properties of space are assigned not by a local coordinates description of the metric tensor, but through a "dual viewpoint", spectrally, in terms of the Dirac operator, hence they continue to make sense on spaces that no longer exist classically. This appears to be a promising approach to reconcile space (no longer defined classically) with the dimensional formulation.

It is important to stress, in this respect, that the formulation of Riemannian spin geometry in the setting of noncommutative geometry, in fact, has already built in the possibility of considering a geometric space at dimensions that are complex numbers rather than integers. This is seen through the dimension spectrum, which is the set of points in the complex plane at which a space manifests itself with a nontrivial geometry. There are examples where the dimension spectrum contains points off the real lines (e.g. the case of Cantor sets), but here one is rather looking for something like a deformation of the geometry in a small neighborhood of a point of the dimension spectrum, which would reflect dimensional regularization. The possibility of recasting the dimensional formulation in the setting of noncommutative geometry may prove very useful in the problem of extending at a fully quantum level the geometric interpretation of the standard model of elementary particle physics provided by noncommutative geometry ([14, [10]).

An important related question, which may be a starting point for such broader program, is to understand the precise relation between the universal singular frame and the local index formula, which in turn may cast some new light on the issue of the relation of the theory of perturbative renormalization illustrated here and noncommutative geometry. Since the local index formula of Connes-Moscovici is closely related to chiral anomalies, a direct comparison with the local index formula will involve a well known problem associated to dimensional regularization in the chiral case, namely the technical issue of how to extend the definition of the product

$$
\gamma_{5}=i \gamma^{0} \gamma^{1} \gamma^{2} \gamma^{3}
$$

of the $\gamma$ matrices, which integer dimension $D=4$ satisfies the Clifford relations $\left\{\gamma^{\mu}, \gamma^{\nu}\right\}=2 g^{\mu \nu} I$, with $\operatorname{Tr}(I)=4$, and anticommutativity $\left\{\gamma_{5}, \gamma^{\mu}\right\}=0$. The $\gamma^{5}$ problem, however, is not considered a serious obstacle to the application of dimensional regularization, as there are good methods to address it ( $c f$. 49, for a recent discussion of this issue). For instance, the $\gamma^{5}$ problem is addressed by the Breitenlohner-Maison approach, in which one does not give an explicit expression for the gamma matrices in complex dimension, but just defines them (and the $\gamma_{5}$ given by (6.1) through their formal properties. In [43, Kreimer described another approach to the problem, in which $\gamma_{5}$ still anticommutes with $\gamma^{\mu}$ but the trace is no longer cyclic, an approach that is expected to be equivalent to the one of Breitenlohner-Maison ( $c f$. 43, $\S 5)$.

Finally, we would like to end on a more speculative tone, by mentioning a very different source for the idea of the existence of a deformation of geometry to nonintegral complex dimensions. In arithmetic geometry, the Beilinson conjectures relate the values of the first non-vanishing derivative at integer points of the motivic $L$-functions of algebraic varieties to periods, namely numbers obtained by 
integration of algebraic differential forms on algebraic varieties (cf. e.g. [42). This process of considering the expansion in a neighborhood of an integer point is reminiscent of the procedure of Dim-Reg, where one considers the Feynman integrals in an infinitesimal neighborhood of the integer dimension $D$. Based on this analogy, it becomes extremely suggestive to imagine that the Beilinson conjectures may be related to a "dimensional regularization of algebraic varieties and periods", and that there may be a geometric interpretation even for the values at non-integer points, in terms of some (noncommutative) geometry in complex dimension.

\section{REFERENCES}

[1] D.V. Anosov, A.A. Bolibruch, The Riemann-Hilbert problem, Aspects of Mathematics Vol.22, Vieweg, 1994.

[2] H. Araki, Expansional in Banach algebras, Ann. Sci. École Norm. Sup. (4) 6 (1973), 67-84.

[3] A. Beilinson, J. Bernstein, P. Deligne, Faisceaux pervers, Astérisque 100 (1982) 5-171.

[4] S. Bloch, Lectures of mixed motives, in "Algebraic geometry-Santa Cruz 1995", pp.329-359, Proc. Sympos. Pure Math., 62, Part 1, Amer. Math. Soc., 1997.

[5] N.N. Bogoliubov, O. Parasiuk, On the multiplication of the causal function in the quantum theory of fields, Acta Math. 97, (1957), 227-266.

[6] N.N. Bogoliubov and D.V. Shirkov, Introduction to the theory of quantized fields, 3rd ed., Wiley 1980.

[7] B. Bojarski, Connections between complex and global analysis: some analytical and geometrical aspects of the Riemann-Hilbert transmission problem. in "Complex analysis", 97-110, Math. Lehrbücher Monogr. II. Abt. Math. Monogr., 61, Akademie-Verlag, 1983.

[8] A.A. Bolibruch, The Riemann-Hilbert problem and Fuchsian differential equations on the Riemann sphere, Proceedings of the International Congress of Mathematicians, Zürich 1994, Birkäuser 1995, 1159-1168.

[9] P. Cartier, A mad day's work: from Grothendieck to Connes and Kontsevich. The evolution of concepts of space and symmetry, Bull. Amer. Math. Soc. (N.S.) 38 (2001), no. 4, 389-408.

[10] A.H. Chamseddine, A. Connes, The spectral action principle. Comm. Math. Phys. Vol.186 (1997), N.3, 731-750.

[11] K.T. Chen, Iterated integrals and exponential homomorphisms, Proc. London Math. Soc. Vol.3 N.4 (1954), 502-512.

[12] K.T. Chen, Iterated integrals of differential forms and loop space homology. Ann. of Math. (2) 97 (1973), 217-246.

[13] J. Collins, Renormalization, Cambridge Monographs in Math. Physics, Cambridge University Press, 1984.

[14] A. Connes, Gravity coupled with matter and the foundation of non-commutative geometry. Comm. Math. Phys. Vol.182 (1996), N.1, 155-176.

[15] A. Connes, D. Kreimer, Hopf algebras, renormalization and noncommutative geometry, Comm. Math. Phys. Vol. 199 (1998) 203-242.

[16] A. Connes, D. Kreimer, Renormalization in quantum field theory and the Riemann-Hilbert problem. I. The Hopf algebra structure of graphs and the main theorem. Comm. Math. Phys. 210 (2000), no. 1, 249-273.

[17] A. Connes, D. Kreimer, Renormalization in quantum field theory and the Riemann-Hilbert problem. II. The $\beta$-function, diffeomorphisms and the renormalization group. Comm. Math. Phys. 216 (2001), no. 1, 215-241.

[18] A. Connes, M. Marcolli, Renormalization and motivic Galois theory, International Math. Research Notices (2004) N.76, 4073-4092.

[19] A. Connes, M. Marcolli, From Physics to Number Theory via Noncommutative Geometry. Part II: Renormalization, the Riemann-Hilbert correspondence, and motivic Galois theory, preprint hep-th/0411114

[20] A. Connes, H. Moscovici, The local index formula in noncommutative geometry, GAFA, Vol. 5 (1995), 174-243.

[21] P. Deligne, Equations differentielles à points singuliers réguliers, Lecture Notes in Mathematics 163, Springer 1970. 
[22] P. Deligne, Catégories tannakiennes, in "Grothendieck Festschrift" Vol.2, pp. 111-195, Progress in Mathematics Vol.87, Birkhäuser, 1990.

[23] P. Deligne, A quoi servent les motifs?, in "Motives" (Seattle, WA, 1991), pp.143-161, Proc. Sympos. Pure Math., 55, Part 1, Amer. Math. Soc., 1994.

[24] P. Deligne, A.B. Goncharov, Groupes fondamentaux motiviques de Tate mixte, math.NT/0302267

[25] P. Deligne, J.S. Milne, , in "Hodge cycles, motives, and Shimura varieties", Lecture Notes in Mathematics, 900, pp.101-228. Springer-Verlag, 1982

[26] M. Demazure, A. Grothendieck, et al. Séminaire Géometrie Algébrique: Schémas en Groupes, Lecture Notes in Mathematics, Vol. 151, 152, 153. Springer, 1970.

[27] S. Doplicher, J.E. Roberts, A new duality for compact groups. Invent. Math. 98 (1989) 157218.

[28] M. Douglas, Dirichlet branes, homological mirror symmetry, and stability, in "Proceedings ICM 2002" Beijing 2002, Vol.III, pp.395-408, Higher Education Press, 2002.

[29] S.I. Gelfand, Yu.I. Manin, Homological algebra, Encyclopaedia of Mathematical Sciences, Vol.38, Springer, 1994.

[30] A. Goncharov, Multiple polylogarithms and mixed Tate motives, math.AG/0103059

[31] A. Grothendieck, Sur la classification des fibrés holomorphes sur la sphère de Riemann, Amer. J. Math. Vol.79 (1957) 121-138.

[32] A. Grothendieck, Esquisse d'un programme, 1984 manuscript, reproduced in "Geometric Galois actions, 1", 5-48, Cambridge Univ. Press, 1997.

[33] A. Grothendieck, Récoltes et Semailles, unpublished manuscript, 1985-86.

[34] K. Hepp, Proof of the Bogoliubov-Parasiuk theorem on renormalization, Comm. Math. Phys. 2, (1966), 301-326.

[35] G. 't Hooft, Dimensional regularization and the renormalization group, Nuclear Physics B, 61 (1973) 455-468.

[36] G. 't Hooft, M. Veltman, Regularization and renormalization of gauge fields Nuclear Physics B, Vol.44, N.1 (1972), 189-213.

[37] S.A. Joni, G.C. Rota, Coalgebras and bialgebras in combinatorics. Stud. Appl. Math. 61 (1979), no. 2, 93-139.

[38] M. Kashiwara, Faisceaux constructibles et systèmes holonômes d'équations aux dérivées partielles linéaires à points singuliers réguliers, Séminaire Goulaouic-Schwartz, 1979-1980, Exp. No. 19, 7 pp., École Polytech., Palaiseau, 1980.

[39] M. Kashiwara, The Riemann-Hilbert problem for holonomic systems. Publ. Res. Inst. Math. Sci. 20 (1984), no. 2, 319-365.

[40] M. Kontsevich, Operads and motives in deformation quantization, Lett. Math. Phys. 48 (1999), no. 1, 35-72.

[41] M. Kontsevich, private communication.

[42] M. Kontsevich, D. Zagier, Periods, in "Mathematics unlimited-2001 and beyond", pp. 771808, Springer, 2001.

[43] D. Kreimer, The role of $\gamma_{5}$ in Dimensional Regularization, hep-ph/9401354

[44] D. Kreimer, On the Hopf algebra structure of perturbative Quantum Field Theory, Adv. Theor. Math. Phys. 2 (1998), no. 2, 303-334.

[45] Lê Dũng Tráng, Z. Mebkhout, Introduction to linear differential systems, Proc. Symp. Pure Math. Vol. 40 (1983) Part 2, pp. 31-63, American Mathematical Society 1983.

[46] M. Levine, Mixed motives, Math. Surveys and Monographs, Vol. 57, AMS, 1998.

[47] Yu.I. Manin, Moduli fuchsiani, Ann. Scuola Norm. Sup. Pisa, Vol.19 (1965) N.3, 113-126.

[48] Yu.I. Manin, Correspondences, motifs and monoidal transformations, Mat. Sb. (N.S.) 77 (119) $1968,475-507$.

[49] C.P. Martín, D. Sánchez-Ruiz, Action principles, restoration of BRS symmetry and the renormalization group equation for chiral non-Abelian gauge theories in dimensional renormalization with a non-anticommuting $\gamma_{5}$, Nucl.Phys. B572 (2000) 387-477.

[50] J. Martinet, J.P. Ramis, Elementary acceleration and multisummability, I, Ann. Inst. Henri Poincaré, Vol.54 (1991) 331-401.

[51] Z. Mebkhout, Sur le problème de Hilbert-Riemann C. R. Acad. Sci. Paris Sér. A-B 290 (1980), no. 9, A415-A417. 
[52] Z. Mebkhout, Sur le problème de Hilbert-Riemann. in "Complex analysis, microlocal calculus and relativistic quantum theory" (Proc. Internat. Colloq., Centre Phys., Les Houches, 1979), pp. 90-110, Lecture Notes in Phys., 126, Springer, Berlin-New York, 1980.

[53] M. van der Put, M. Singer, Galois theory of linear differential equations, Springer 2002.

[54] M. van der Put, Differential Galois Theory, Universal Rings and Universal groups, in "Differential Algebra and Related topics", Editors Li Guo, Phyllis Cassidy, William F. Keigher, William Sitt. World Scientific 2002.

[55] G.C. Rota, Hopf algebra methods in combinatorics, in "Problèmes combinatoires et théorie des graphes" (Colloq. Internat. CNRS, Univ. Orsay, Orsay, 1976), pp. 363-365, Colloq. Internat. CNRS, 260, CNRS, Paris, 1978.

[56] N. Saavedra Rivano, Catégories Tannakiennes. Lecture Notes in Mathematics, Vol. 265. Springer-Verlag, 1972.

[57] W. Schmitt, Hopf algebras in enumerative combinatorics. in "Gian-Carlo Rota on combinatorics", 345-356, Contemp. Mathematicians, Birkhäuser, 1995.

[58] J.P. Serre, Motifs, in "Journées Arithmétiques, 1989" (Luminy, 1989). Astérisque No. 198-200 (1991), 11, 333-349 (1992).

[59] J.P. Serre, Propriétés conjecturales des groupes de Galois motiviques et des representations l-adiques, Proc. Symp. Pure Math. Vol. 55 (1994) Part I, 377-400.

[60] M. Veltman, Perturbation theory and relative space, Acta Physica Polonica B Vol.25 N.10 (1994) 1399-1411.

[61] V. Voevodsky, Triangulated categories of motives over a field in "Cycles, transfer and motivic homology theories, pp. 188-238, Annals of Mathematical Studies, Vol. 143, Princeton, 2000.

[62] W. Zimmermann, Convergence of Bogoliubov's method of renormalization in momentum space, Comm. Math. Phys. 15, (1969), 208-234.

A. Connes: Collège de France, 3, Rue D'Ulm, Paris, F-75005 France

E-mail address: alain@connes.org

M. Marcolli: Max-Planck Institut für Mathematik, Vivatsgasse 7, Bonn, D-53111 GerMANY

E-mail address: marcolli@mpim-bonn.mpg.de 